\title{
Mechanical and Energy Absorption Properties of Functionally Graded Lattice Structures based on Minimal Curved Surfaces
}

\section{Xiangyu Ma}

Chongqing University

David Zhengwen Zhang ( $\square$ zhangzw@cqu.edu.cn )

University of Exeter https://orcid.org/0000-0002-1561-0923

Miao Zhao

Chongqing University

Junjie Jiang

Zhengzhou University of Light Industry

\section{Fangqiong Luo}

Chongqing University

Hailun Zhou

Zhengzhou University of Light Industry

\section{Research Article}

Keywords: Functional graded structure, Triply periodic minimal surface, SLM, Finite element analysis, Compression behavior, Energy absorbing

Posted Date: May 5th, 2021

DOl: https://doi.org/10.21203/rs.3.rs-464943/v1

License: (c) (1) This work is licensed under a Creative Commons Attribution 4.0 International License. Read Full License

Version of Record: A version of this preprint was published at The International Journal of Advanced Manufacturing Technology on September 12th, 2021. See the published version at https://doi.org/10.1007/s00170-021-07768-y. 


\title{
Mechanical and energy absorption properties of functionally graded lattice structures
}

\section{based on minimal curved surfaces}

\author{
Xiangyu Ma ${ }^{\mathrm{a}, \mathrm{b}}$, David Z. Zhang ${ }^{\mathrm{a}, \mathrm{b}, \mathrm{c}, *}$, Miao Zhao ${ }^{\mathrm{a}, \mathrm{b}}$, Junjie Jiang ${ }^{\mathrm{d}}$, Fangqiong Luo ${ }^{\mathrm{a}, \mathrm{b}}$, Hailun Zhou ${ }^{\mathrm{c}}$ \\ a State Key Laboratory of Mechanical Transmissions, Chongqing University, Chongqing, 400044, China \\ b Chongqing Key Laboratory of Metal Additive Manufacturing (3D Printing), Chongqing University, Chongqing, 400044, China \\ c College of Engineering, Mathematics and Physical Sciences, University of Exeter, North Park Road, Exeter EX4 4QF, UK \\ d Mechanical and Electrical Engineering College, Zhengzhou University of Light Industry, Zhengzhou 450002, China \\ * Corresponding author. E-mail address: zhangzw@cqu.edu.cn (D.Z. Zhang).
}

Abstract: Compared with uniform structures, functionally graded lattice structures can control mechanical properties through varying structure and volume fraction. In this study, a three-period minimal surface method was used to generate functional lattice structure with linear or quadratic function (LF or QF) gradient strategy in the forming direction, and the samples were fabricated by selective laser melting (SLM) using Ti-6Al-4V metal powder. The mechanical properties, deformation behavior, and energy absorption performance of graded lattice, LF and QF I-Wrapped Package (IW-P) lattice structures were systematically investigated through experiment and finite element analysis (FEA). Based on the experiment and numerical simulation results, the LF lattice structure shows higher elastic modules and yield strength during small strain period. And the merits of performance increased layer-by-layer under large strain. Additionally, the simulation results based on Johnson-Cook and failure model show that this model can reflect structural compression deformation behavior and mechanical performance prediction. Furthermore, the elastic modulus of LF lattice structure is higher than uniform lattice structures by nearly $61.52 \%$ under the same volume fraction. Thence, the LF or QF lattice structures have better support performance under small strain and stronger energy absorption capacity under large strain with the same volume fraction compared with other lattice structures, which shows superior potential to be applied to manufacture protective devices or vibration damping devices.

Keywords : Functional graded structure; Triply periodic minimal surface; SLM; Finite element analysis; Compression behavior; Energy absorbing. 


\section{Introduction}

In the course of engineering design, manufacturing and technological innovation, nature has been a constant source of inspiration. As is known to all, those conflicts which are key challenges for the enhancement of the mechanical performance of structures restricted by a series of contradictions among different properties can be easily solved in nature[1]. Inspired by the structures of wheat awn and softwood branches[2], turtle rib[3], shark denticle[4], bamboo[5] and other natural structures, many scientific research teams have been trying to find an optimal method from nature to achieve higher performance requirements[6-9]. Function graded structure (FGS) is one of the design methods derived from nature, and has been widely used in various fields, such as heat dissipating[10, 11], energy absorption[12-15], optoelectronic and thermoelectric[16], biomedical prosthetic device[17-21] machinery and equipment application[22, 23].

Although the idea of graded materials was firstly used by Japanese engineering to reduce thermal stress from the high temperature of the metal and ceramic interfaces[24], these materials have been used to control deformation, pressure and wear with time passing by. According to the performance of gradient materials in different working environment or functional requirements, gradient materials can be further divided into functionally gradient materials and functionally gradient structures, which are not new as there are series of examples in human body parts, such as bones, skin and specific dental crowns[25]. At present, the research contents of functional gradient structure mainly include design method, performance research and application for special requirement. Among them, functional design method is a more advantageous design method since it can govern the morphological parameters such as pore size and volume fraction of lattice structures. Some scholars also have been attempting to design the FGS through designing CAD model[26] and biological reverse[27]. And many performances of lattice structure have been discussed, such as statics[28-31], dynamics and fatigue characteristics[32]. Applications for special needs have been described previously and will not be repeated here. Based on the previous description, FGS has been fully discussed. In view of the tremendous advantages of FGS, many research teams strive to improve and develop their production methods for expanding the field of application of FGS aiming to meet the requirement of fourth industrial revolution.

Currently, as a function-driven design method, triply periodic minimal surfaces (TPMS) are 
mathematically defined structures that repeat in three dimensions with zero mean curvatures and large surface areas, which can be easy found in nature[33]. Among these, this mathematical approach is characterized by a cubic symmetry and has interconnected internal void regions. These characteristics are beneficial for the design lightweight scaffolds, where the designer can specify the volume fraction and stiffness of the structure, and also for functional graded structure[34]. In recent years, additive manufacturing (AM) methods have been put in place in order to make significant impact on the development of FGS and replace the conventional metal production model using sophisticated layer-layer-size machinery[25]. Various current works have been explored in TPMS lattice structures based on additive manufacturing technology. A study of the primitive TPMS scaffold made by AM showed higher ultimate stress, more total energy absorption per unit volume and higher energy efficiency, which means a widespread application of comparatively high allowable stress in the future[28, 30,35]. The same work analyzed the elastic properties and yield stress states of the structure through the finite element analysis method. In another work, cell size gradient porous scaffolds based on TPMS are becoming an attractive candidate for bone grafts[36]. Other studies analyzed the energy absorbed during compressive loading from three types of TPMS made of Ti alloy: gyroid, diamond, and Primitive[28, 30, 36, 37].

In spite of these, there are still different FGS with different materials or structures being put forward to meet the performance requirements of different workplaces, making huge contributions to the application of FGS, such as the lattice structure with variable volume fraction[28, 37], the gradient lattice structure with variable unit cell size[26, 28] and Multi-material gradient structure[38]. Moreover, the mathematical modelling, the prediction and determination of mechanical properties and the analytical models of FGS mechanics are especially essential for the future direction[25]. To acquire a lattice structure with better carrying capacity and energy absorption, a new lattice gradient strategy based on TPMS was proposed in this paper. In order to verify this gradient strategy, a uniform lattice structure, a density gradient lattice, LF and QF with the same volume fraction were designed. Furthermore, the mechanical properties obtained from experimental and numerical simulation data of these four lattice structures were compared, which involved deformation behaviors, energy absorption performance and failure mechanisms of the uniform IW-P, FGS IW-P, LF IW-P and QF IW-P fabricated by AM with Ti-6Al-4V. In this article, the samples were fabricated by SLM technology include a uniform lattice structure with volume 
fraction of $20 \%$, a graded structure with $20 \%$ gradient of a volume fraction and a lattice structure with a volume fraction of $20 \%$ with a dimensional linear gradient or a quadratic gradient in the manufacturing direction. The morphological characteristics, mechanical properties, and energy absorption capability of the fabricated lattice structures were investigated. Through the quasi-static compression experiment and finite element analysis with the Johnson-Cook plasticity model and damage model, the mechanical properties and energy absorption characteristics of the lattice structure have been studied in detail.

\section{Materials and methodology}

\subsection{Design of graded I-WP lattice structures}

The network based I-WP structure can be mathematically defined by TPMS equations using implicit methods. The I-WP lattice structure is generated from the following mathematical functions:

$$
\begin{gathered}
S_{W}(x, y, z)=\cos (2 a x)+\cos (2 a y)+\cos (2 a z)+k[\cos (a x) \cos (a y)+\cos (a y) \cos (a z)+ \\
\cos (a z) \cos (a x)]+d^{\%} \leq t^{\%}
\end{gathered}
$$

Where the parameter $d^{\%}$ is the volume fraction of network based I-WP structures, which is controlled by the width of the matrix phase region $t^{\%}$. Where the parameter a is the TPMS function periodicities to control the unit size in $\mathrm{x}, \mathrm{y}$ and $\mathrm{z}$ directions, which is defined by:

$$
\mathrm{a}=2 \pi / L
$$

Where $\mathrm{L}$ is the length of a lattice unit in $\mathrm{x}, \mathrm{y}$ and $\mathrm{z}$ direction.

The volume fractions $d^{\%}$ of the unit cell of network based I-WP structure is defined by the following:

$$
d^{\%}=\frac{\iiint\left(S_{W}(x, y, z) \leq t^{\%}\right) \cap(|x| \leq L / 2) \cap(|y| \leq L / 2) \cap(|z| \leq L / 2) d x d y d z}{L^{3}}
$$

Then, networks I-WP lattice structures with predefined volume fractions $\left(\rho^{\%}\right)$ were generated to develop the relationship between the volume fraction $\left(\rho^{\%}\right)$ and $t \%$, as shown in

Fig.1 


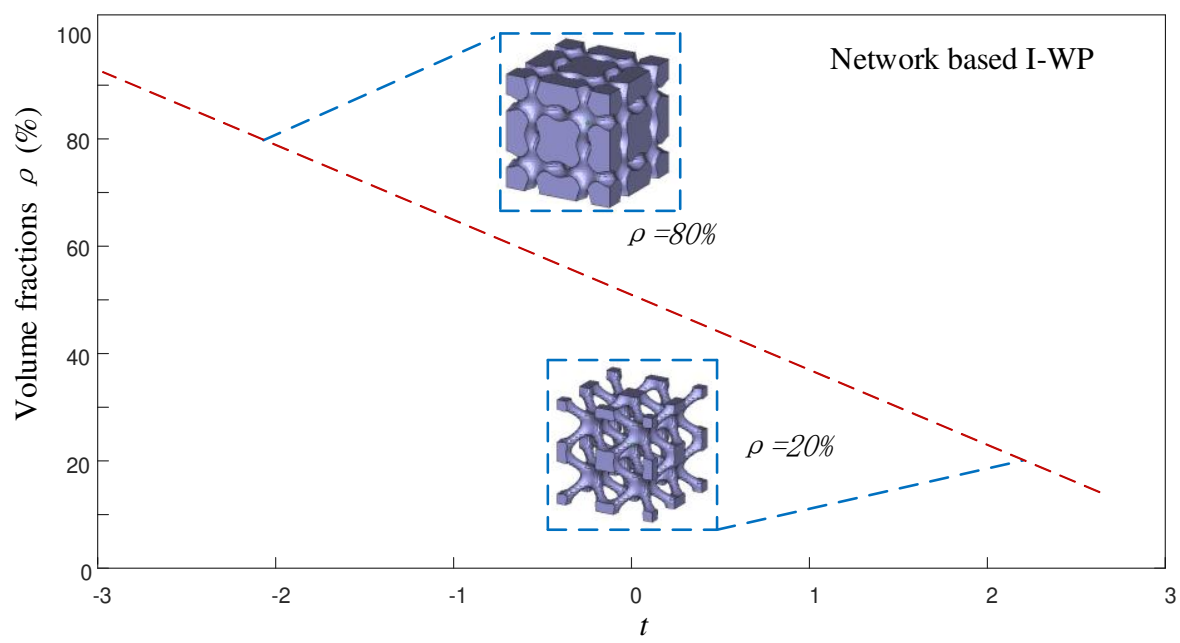

Fig.1 The functional relationship between $\mathrm{t}$ and $\rho$ of network based I-WP structures.

In this study, the design samples had an overall dimension of $20 \mathrm{~mm} \times 20 \mathrm{~mm} \times 20 \mathrm{~mm}$ corresponding to $4 \times 4 \times 4$ lattice unite along each direction, which was generated by setting the parameters $\mathrm{a}=\pi / 2$ and $\mathrm{x}, \mathrm{y}, \mathrm{z} \in[0,20]$. Both lattice structures were achieved with an average volume fraction of $20 \%$. For the uniform IW-P lattice structure, the $t^{\%}=2.169$, which is shown in Fig.2(a). As shown in Fig.2(b), the graded lattice structures, and the volume fraction of gradient IW-P model change from $10 \%$ to $30 \%$ in $z$ direction through setting the parameter by formula (4). For the LF IW-P lattice structures, the volume fraction was setting $20 \%$ by changing the cell size of unit cells through formula (5). And the QF IW-P lattice structure, the volume fraction was setting $20 \%$ through changing the size of unit cells in the forming direction by formula (6): The CAD models were performed by a MATLAB code to generate the STL files and processed in Magics software.

$$
\begin{aligned}
& t^{\%}(z)=0.043123 z+1.597 \\
& t^{\%}=0.008333 z+2 \\
& t^{\%}=0.001 z^{2}+0.00125 z+2
\end{aligned}
$$




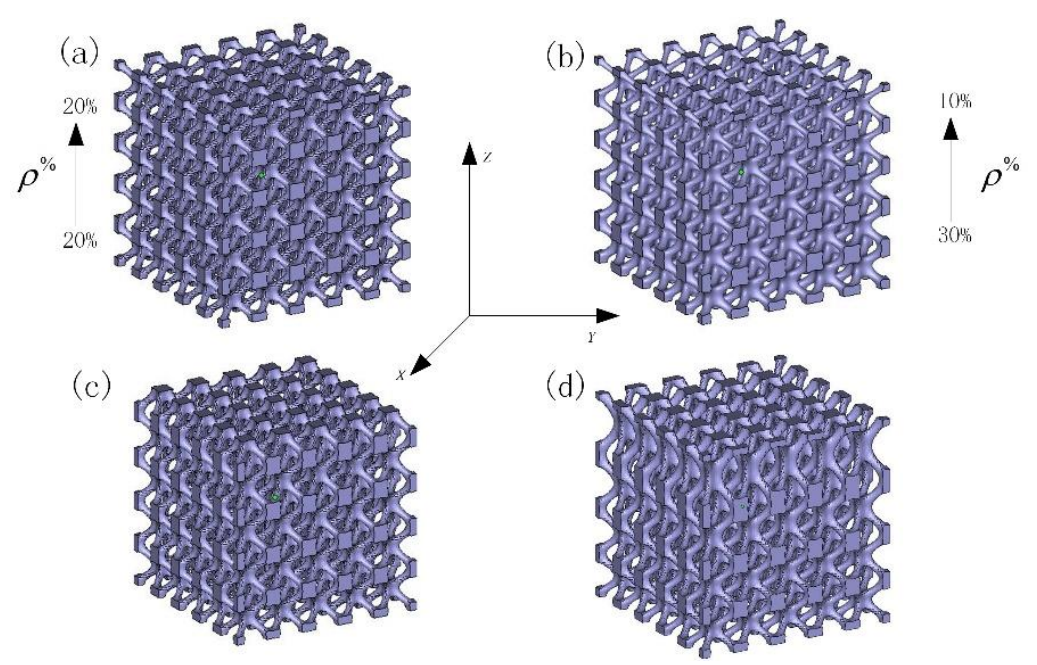

Fig.2 Three-dimensional models of (a) Uniform IW-P; (b)Graded IW-P;

(c) LF IW-P and (d) QF IW-P lattice structures

2.2 Materials and manufacture

\subsubsection{Materials}

The scanning electron microscope (SEM) micrographs of the commercial TC4 powder used in this study are demonstrated in Fig.3. The SEM micrograph shows a nearly spherical shape with smooth surfaces (Fig.3 (a)), leading to a good flowability for SLM process. A narrow particle size distribution was confirmed by laser light diffraction, as shown in Fig.3(b). All of these features provide a guarantee for precision shaping. The particle size exhibited a narrow distribution between $25.0 \mu \mathrm{m}$ and $52.3 \mu \mathrm{m}$ with an average diameter of $35.4 \mu \mathrm{m}$.
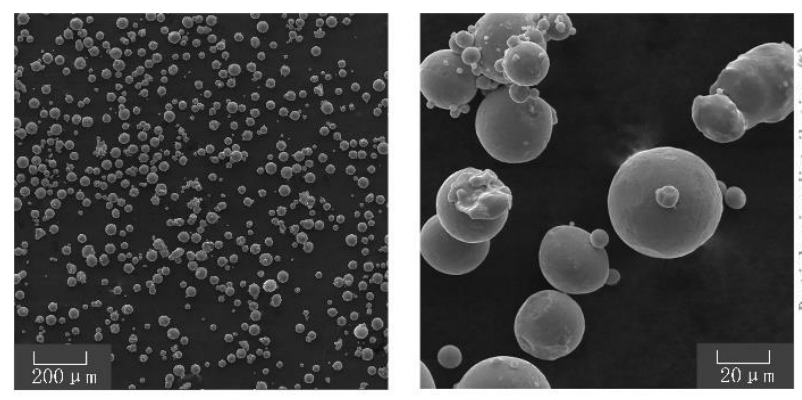

(a) SEM micrograph

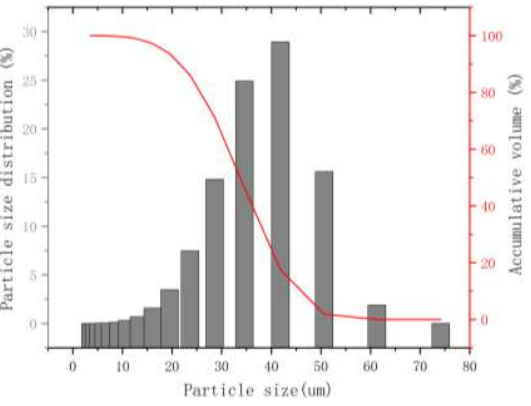

(b) particle size distribution

Fig.3 (a) SEM micrograph and (b) particle size distribution of TC4 powder

\subsubsection{Manufacture}

The experimental samples of each TPMS lattice structure were manufactured by SLM using 
an EOSINT-M280 (EOS GmbH, Germany). The used processing parameters were as follows: laser power of $175 \mathrm{~W}$, laser beam diameter of $0.1 \mathrm{~mm}$, layer thickness of $0.03 \mathrm{~mm}$, hatch spacing of $0.1 \mathrm{~mm}$ and scanning speed of $1250 \mathrm{~mm} / \mathrm{s}$. The laser melting process occurs in an argon gas environment with an $\mathrm{O}^{2}$ content less than $0.1 \%$ and the building substrate heating temperature was maintained at $35^{\circ} \mathrm{C}$. Then, the fabricated samples were removed from base plate by wire electrical discharge machining. The graded lattice structures were built with the same processing parameters and the manufactured samples are shown in Fig.6.

2.3 Finite element modelling

\subsubsection{Finite element mesh construction and convergence}

Aiming to predict the deformation behavior and mechanical properties of these lattice structures, the finite element analysis was carried out using ABAQUS/explicit 2019. Both element types of the uniform, graded, LF and QF lattice structure models are linear hexahedron meshes (C3D8R) which were generated by specifying the coordinates of nodal points in MATLAB R2016a[39], with the whole size of each FE model setting to $20 \mathrm{~mm} \times 20 \mathrm{~mm} \times 20 \mathrm{~mm}$ showed in Fig.4. Then, the upper plate moved downward in the direction of the red arrow, and the bottom plate stayed still, which is indicated by a brown straight. Additionally, convergence studies with a range of mesh numbers were conducted to gain a satisfactory mesh size, as shown in Fig.5, and roughly 2480000 hexahedral elements were produced for each model.

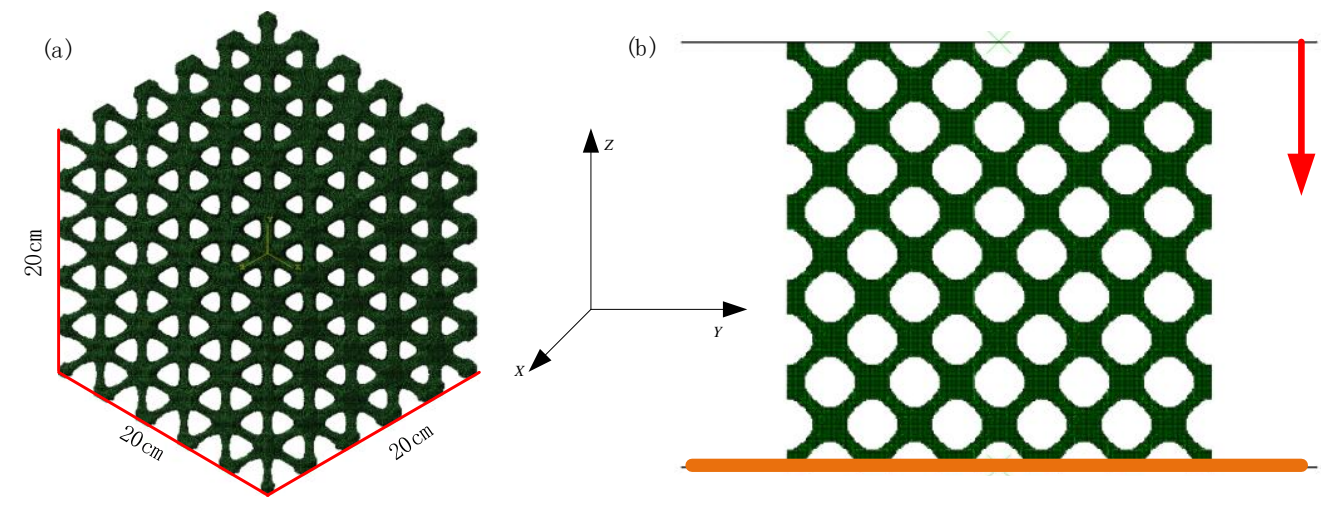

Fig.4 The FE models of hexahedral meshes:

(a) Uniform IW-P (with 20\% volume fraction) based FE model; (b) the boundary condition in FEA 


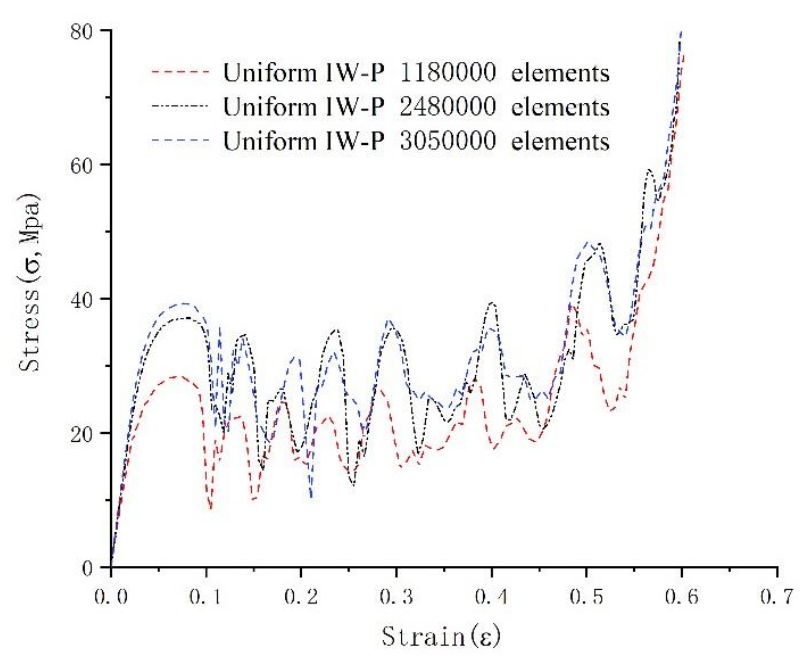

Fig.5 Convergence of stress-strain curves corresponding to the three mesh numbers for the W20 structure.

\subsubsection{Johnson-Cook finite element analytical models}

Johnson-Cook plastic and damage models, which are usually used to research the dynamic mechanical response of materials[40], were implemented into FEA to precisely simulate the plastic deformation and failure behaviors of the lattice samples. According to the model, the yield stress can be expressed as follows:

$$
\sigma_{S}=\left[A+B\left(\varepsilon_{e}\right)^{n}\right] \cdot\left[1+C \ln \left(\varepsilon^{p} / \varepsilon^{0}\right)\right] \cdot\left[1-T^{m}\right]
$$

Where, $\mathrm{A}, \mathrm{B}, \mathrm{C}, \mathrm{n}$ and $\mathrm{m}$ are the model constants based on the flow stress data gained from mechanical tests. $\mathrm{n}$ is the strain hardening exponent, and $\mathrm{m}$ is the temperature exponent. $\sigma_{S}$ is the equivalent stress, $\varepsilon_{e}$ is the equivalent plastic strain, $\varepsilon^{p}$ is the equivalent plastic strain rate, $\varepsilon^{0}$ is the reference equivalent plastic strain rate, $\mathrm{T}$ is the temperature during manufacturing.

Aiming to evaluate the fracture behavior of lattice structure, the Johnson-Cook damage model was used, which is expressed as [39]:

$$
\varepsilon_{f}=\left[D_{1}+D_{2} \exp \left(D_{3} \sigma^{*}\right)\right] \cdot\left[1+D_{4} \ln \left(\dot{\varepsilon}^{p} / \dot{\varepsilon}^{0}\right)\right] \cdot\left[1+D_{5} T^{m}\right]
$$

Where, $\varepsilon_{f}$ is the fracture strain, $D_{1}, D_{2}$ and $D_{3}$, are damage constant parameters related to the relationships between failure strain rate and temperature, $D_{4}$ and $D_{5}$ are constants determined by strain rate and temperature, respectively, and $\sigma^{*}$ is stress triaxiality defined as the ratio of hydrostatic stress and the equivalent stress. Given that the experiments were performed under the constant strain rate at room temperature, and consequently, $C, m, D_{4}$ and $D_{5}$ were ignored. These mentioned parameters of Johnson-Cook models with TC4 referred to previous researches[39, 41], as shown in 
Table1. All simulation was performed on an Intel(R) Xeon(R) Silver 4112 CPU @2.60GHz 2.59GHz with 64GB RAM.

Table1 Johnson-Cook model constants of TC4

\begin{tabular}{cccccc}
\hline A(Mpa) & B(Mpa) & $\mathrm{n}$ & $\mathrm{D} 1$ & $\mathrm{D} 2$ & $\mathrm{D} 3$ \\
\hline 1567 & 952 & 0.4 & -0.09 & 0.25 & -0.5 \\
\hline
\end{tabular}

2.3.3 Compressive experimental procedures

All the lattice structures were examined on the testing machine (CMT5105,Shenzhen Wance Testing Machine Co., Ltd Shenzhen, China) with uniaxial quasi-static compressive tests with $100 \mathrm{KN}$ load cell, and the constant stressing rate was set as $1 \mathrm{~mm} / \mathrm{min}$, accompanied by a video camera recording the deformation behaviors for each sample. To reduce errors, Uniform IW-P, Graded IW-P, LF IW-P and QF IW-P lattice structures were tested in triplicate. Then frames of videos correlated with strain were extracted to exhibit their failure behaviors. The stress-strain curve was the experimental result obtained under normal atmospheric condition.

\section{Results and discussion}

\subsection{Structural characteristics}

The SLM-processed lattice samples are shown in Fig.6, and the Uniform IW-P, Graded IW-P, LF IW-P and QF IW-P structures were found to have a continuous graded thickness that conformed to the design, which was consistent with the design ones, as shown in Fig.6. In terms of actual quality and geometric dimensions, there will be a certain error between the manufactured samples and the design values, just as described in other documents[42]. Herein, the size and quality of the manufactured samples of the uniform IW-P lattice structure were given, as shown in Table2. As seen in Table2, the dimensions along $\mathrm{x}$ and $\mathrm{y}$ directions in SLM-built samples were higher than in the designed samples, which keep up with other early experimental studies[41]. It is patently obvious from Table2 that the measured mass of lattice samples was a little higher than the designed mass, which is principally generated by the partially-melted powder particles on the lattice surfaces, discovered in the high magnification SEM micro graph in Fig.7. Moreover, it is noteworthy that the disparity between manufactured mass and designed mass was also influenced by the grade in the 
lattice[43], the accuracy of STL models[44], and the porosities of built samples[45]. However, these are not the focus of this paper.

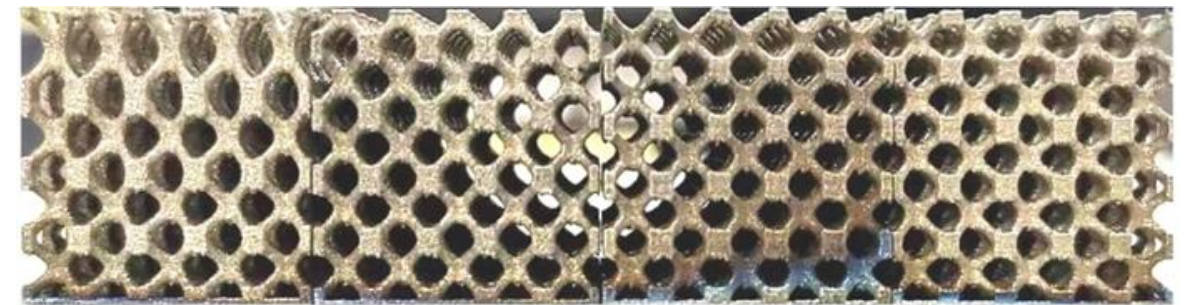
(a)
(b)
(c)
(d)

Fig.6 (a)QF IW-P; (b) LF IW-P; (c) Graded IW-P; (d) Uniform IW-P

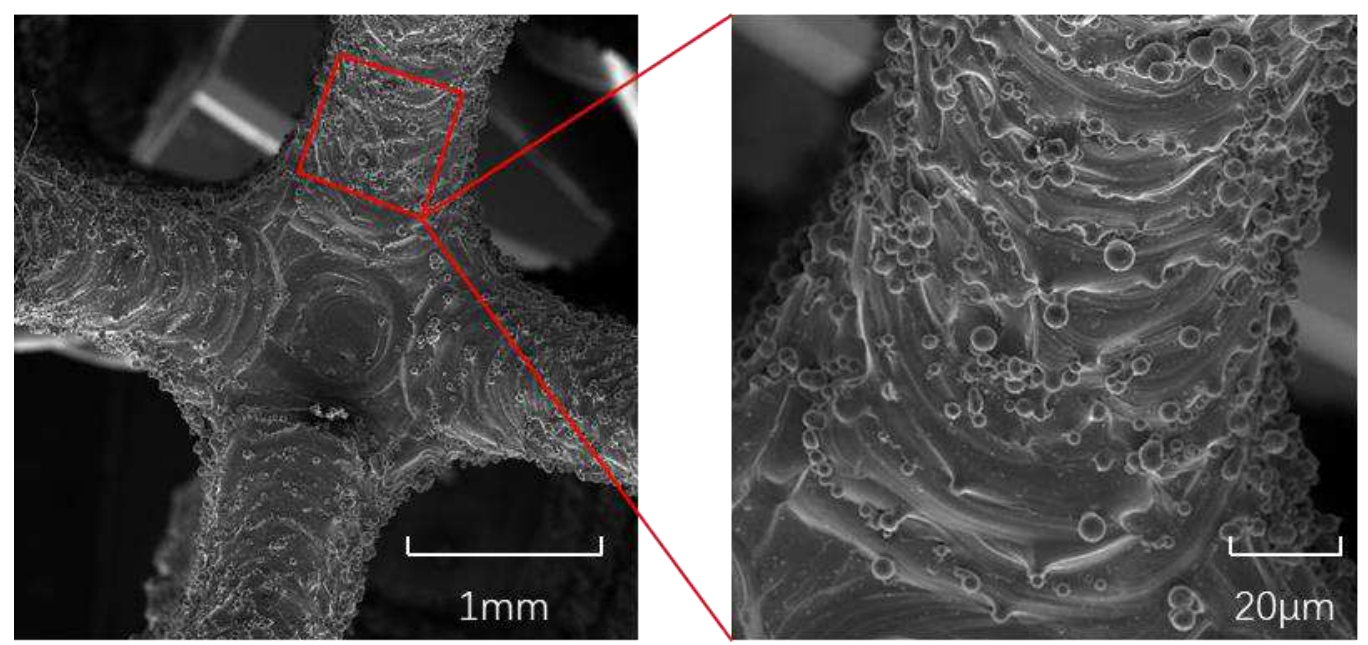

Fig.7 SEM morphology of uniform IW-P structure's surface

Table2 Measured characteristics of uniform IW-P lattice samples.

\begin{tabular}{cccc}
\hline Samples & $\begin{array}{c}\text { Measured dimensions } \\
\mathrm{X} \times \mathrm{Y} \times \mathrm{Z}\left(\mathrm{mm}^{3}\right)\end{array}$ & Measures mass $(\mathrm{g})$ & Designed mass $(\mathrm{g})$ \\
\hline Uniform IW-P & $20.10 \mathrm{~mm} \times 20.18 \mathrm{~mm} \times 19.83 \mathrm{~mm}$ & 7.8161 & 7.08 \\
\hline
\end{tabular}

\subsection{Compressive deformation mechanism}

To acquire mechanical properties of the lattice structure intuitively and qualitatively, the compression research of these lattice structures was studied. In this section, the experimental compression response curves and compression deformation of lattice structures are mainly discussed, which would provide research data for posterior dissection of bearing capacity and energy absorption characteristics.

The stress-strain curves of the experiment and numerical simulation were compared in Fig.8. 
From that, there are three stages in the deformation of the lattice structure, including the linear elastic stage, the elastic-plastic stage and the densification stage in all curves, which are identical to previous researches[46]. Stresses of all the curves increase with the strain increased in the initial of compression and then drop rapidly, which depend on the types of material, and significantly differ from those plastic materials whose stress plateaus exhibit long plateaus without large stress drops, such as $\mathrm{Cu}-\mathrm{Cr}-\mathrm{Zr}[29]$.

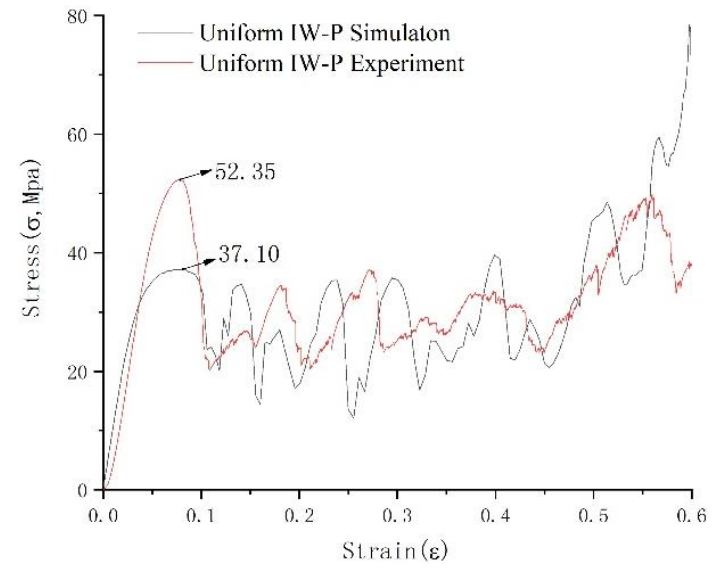

(a) Uniform IW-P

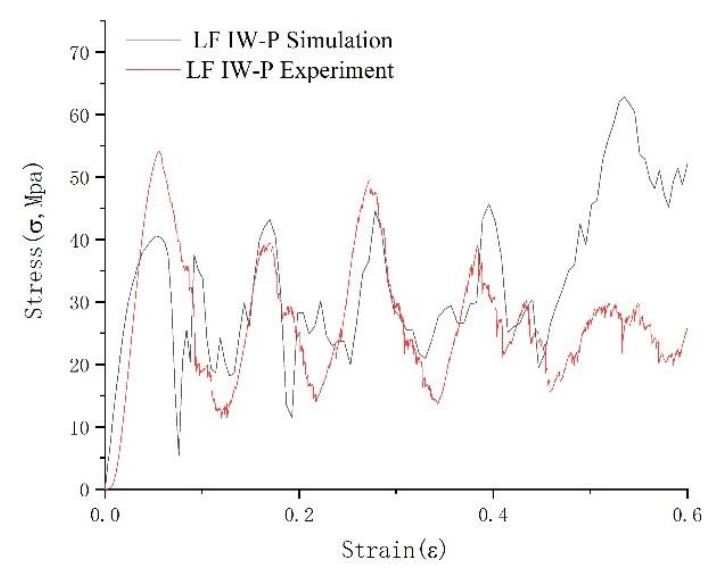

(c) LF IW-P

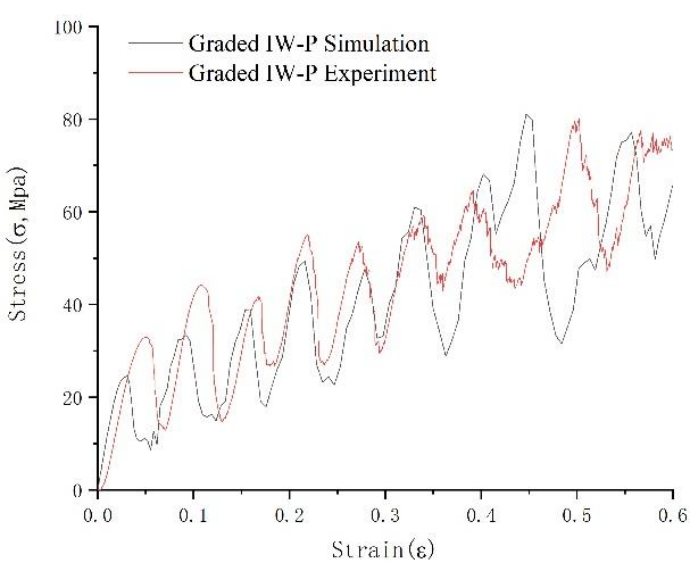

(b) Graded IW-P

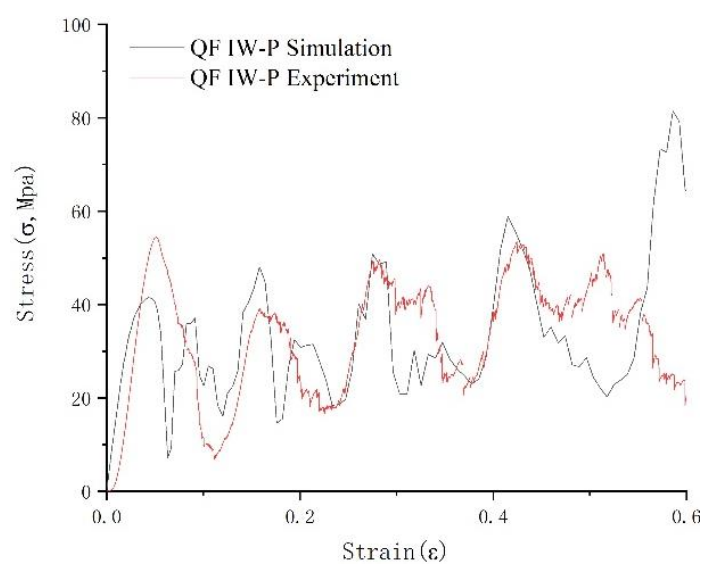

(d) QF IW-P

Fig.8 Simulation and experimental curve comparison of four lattice structures

As shown in Fig.8(a), the stress-strain curve of simulation for uniform IW-P structure demonstrated that the peak of the experimental is ahead of the simulation result from the second peak, and then the deviation between experiment and simulation narrowed gradually with at postyield stage. Eventually, the maximum stress of the compression experimental result is $41 \%$ higher than the initial peak stress of the simulation analysis result with the same trend, as shown in Fig.3, which is a beneficial result for predicting the deformation response at the post-yield stage. 
Nonetheless, the elastic modulus was lower than that in the experimental and the yield stress was higher, as shown in Table3. This deviation was mainly due to the difference in volume fraction between simulation model and as-manufactured samples, especially caused by the shape precision of the simulation model and the voxel-based hexahedron meshes.

For the graded IW-P samples, following the initial stress peak, a significant drop in stress values was surveyed in Fig.8(b), due to the simulation of structural fracture by using the Johnson-Cook damage model. Then followed a linear elastic strain stage where the slope of the stress-strain line determined the elastic modulus of this lattice structure. Thereafter, the stress-strain curves entered the post-yield regions, and it is noted that with every $5 \%$ to $10 \%$ increase in strain, there is an alternating phenomenon of peaks and valleys due to brittle collapse and overloading. The variation trend of the stress-strain cure showed a good agreement with that of the experimental. Nevertheless, there is a larger deviation between the simulation results and the experimental results, which is related to the different amounts of tiny fractures of uniform IW-P samples that flew out during the process of compression simulation and experiment, as shown in Fig.11. This would lead to stress variation owing to the different volume fraction of the remained structures. Additionally, these differences could be exaggerated along with the accumulative layer-by-layer fracture.

The failure form of the LF IW-P structure was described in Fig.8(c). And the simulation stressstrain curve of the lattice structure with the linear function gradient strategy in the building direction maintains a good corresponding relationship with the experimental results. Particularly, the peak stress is $33.2 \%$ higher than the simulated analysis with a certain strain of 0.1 , and the LF structure shows a form of destruction layer-by-layer from bottom to top with the strain increased. However, the stress peak of layer-by-layer is lower than the peak value of the simulation, which could be explained by the factors such as materials properties and flying debris, as shown in Fig.9. Specifically, the material parameters in the Johnson-Cook model are based on the forging material, which is different from the ones of the sample formed by SLM. At the same time, the amount of material debris that failed during the simulation compression process is not consistent with the actual compression process. As shown in Fig.8(d), the stress-strain curve of the QF lattice structure simulation maintains a good trend with the experimental strain-stress, which has a similar trend to the LF structure in Fig.8(c) due to they were the same type of gradient structure. 


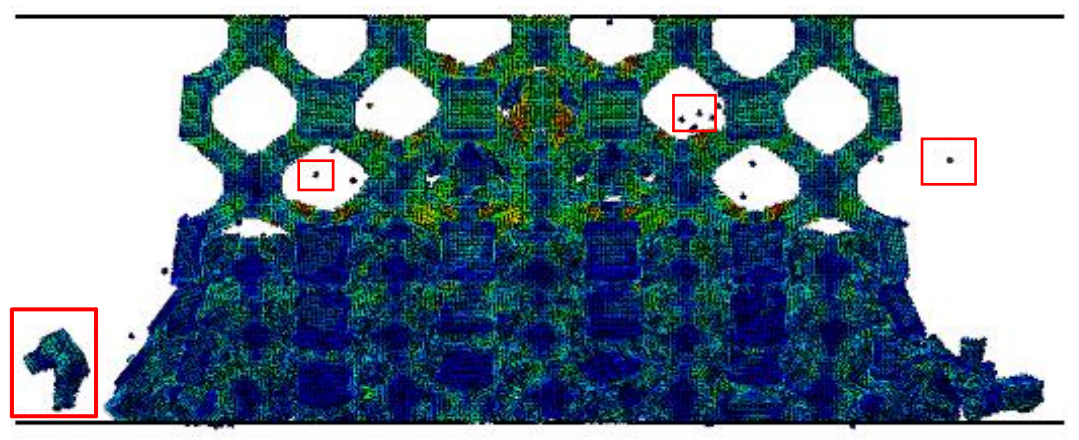

Fig.9 Schematic diagram of LF IW-P lattice structure simulation analysis fragments

Mechanical properties of lattice structure could be intuitively and qualitatively examined through deconstructing the compression response. In order to further analyze and compare the mechanical properties of the four designed structures with the same volume fraction, the stressstrain curves of uniform, graded, LF and QF lattice structures are demonstrated in Fig.10. From that, the mechanical properties of LF IW-P are consistent with that of QF IW-P lattice structure. For example, the compressive strengths of the two structures are 54.80Mpa and 54.33Mpa respectively, which are slightly different from each other. On the other hand, the strain corresponding to the compaction of the two structures is almost at 0.8 , which is caused by the same gradient change strategy in the forming direction based on TPMS. In the strain range of $\varepsilon=0.1-0.7$, both structures show a zigzag curve, which owns to the brittle material properties of TC4. It is observed that the peak value of the line-lattice structure is slightly higher than that of the QF IW-P structure in the first half of the yield stage $(\varepsilon=0.1-0.2)$. This is because the gradual change from the bottom to the top in a linear function and a quadratic function, respectively, and the top face of the quadratic lattice structure has larger pores. 


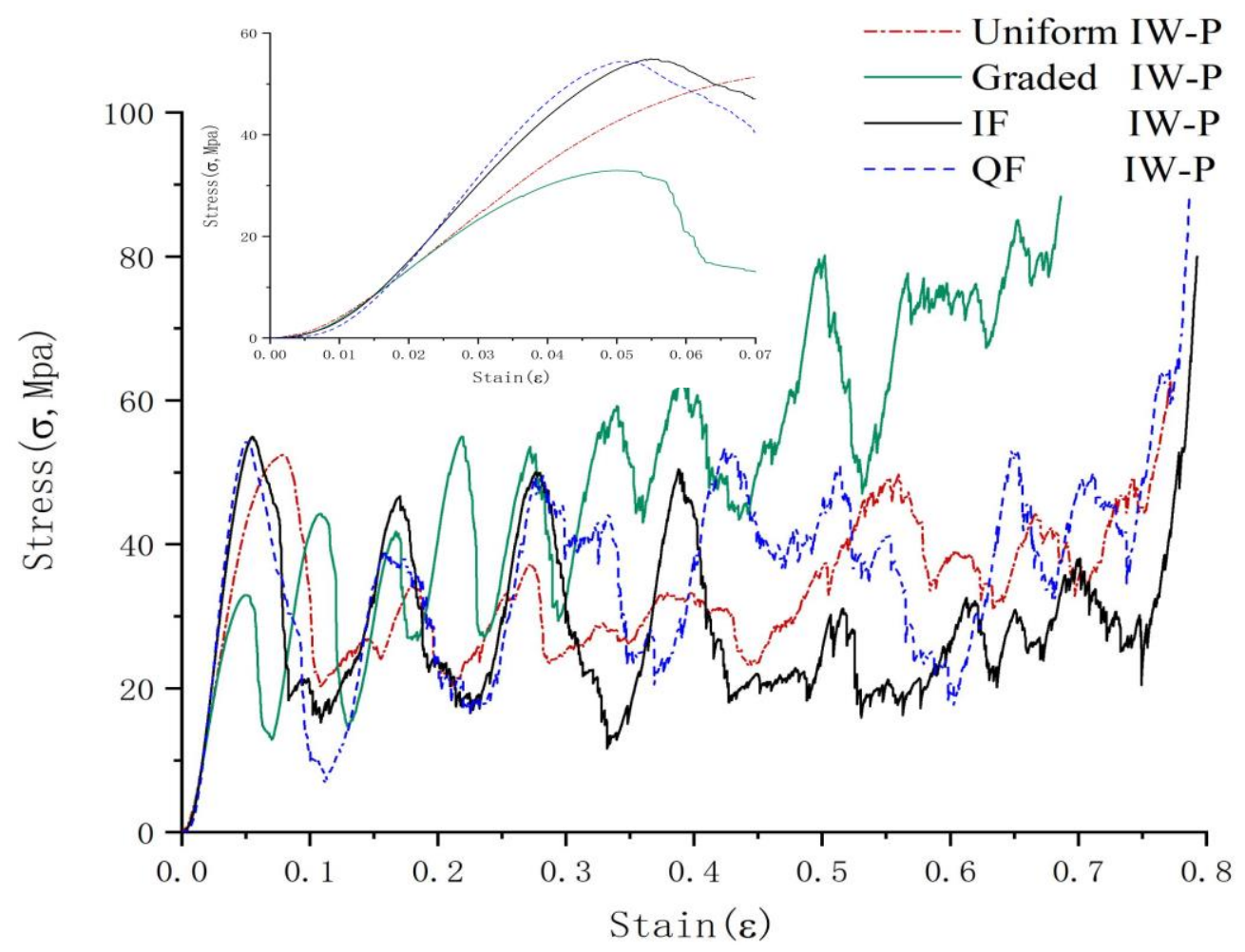

Fig.10 The comparison with experimental results of lattice structure

It is worth noting that, compared with the uniform lattice structure, the gradient lattice structure with a linear function of forming direction has better mechanical properties. For example, the compressive strength of the LF IW-P lattice structure is $4.6 \%$ higher than that of the uniform lattice structure. Furthermore, the LF lattice structure maintains a relatively stable mechanical property of $50 \mathrm{MPa}$ and the uniform lattice structure floats at about $35 \mathrm{MPa}$ in the strain range of 0-0.5. Compared with lattice structures with volume fraction from $10 \%$ (top) to $30 \%$ (bottom), lattice structures with linear function gradient change in forming direction have better mechanical properties within the range of 0-0.3 strain. However, the stress of LF lattice structure is obviously lower than that of QF lattice structure at the value of strain 0.5 , which indicates that the former lattice structure has more stable mechanical properties than that of latter lattice structure, and it has a good reference value in the application of lattice structure design.

Table3 Comparison of compressive strength and stress drop coefficient of four lattice structures

\begin{tabular}{ccccc}
\hline \multirow{2}{*}{ Structure type } & \multicolumn{2}{c}{ First peak (Mpa) } & \multicolumn{2}{c}{ First bottom (Mpa) } \\
\cline { 2 - 5 } & Sim & Exp & Sim & Exp \\
\hline Uniform Structure & 37.10 & 52.35 & 20.13 & 20.30 \\
Graded Structure & 24.61 & 32.96 & 8.61 & 12.93 \\
\hline
\end{tabular}




\begin{tabular}{rrrrc}
\hline LF Structure & 40.57 & 54.05 & 5.37 & 11.44 \\
QF Structure & 41.52 & 54.45 & 7.18 & 6.87 \\
\hline
\end{tabular}

As exhibited in Fig.11, the difference among these four types of structural failure could be noted. For instance, $\mathrm{X}$-shaped destruction bands would appear during the compression process of uniform IW-P structure in the entire structure with a certain amount of strain $(\varepsilon=0.2)$. Nevertheless, during the compression experiment, the structure showed shear failure in the direction of $45^{\circ}$, which is consistent with the previous research[47]. Furthermore, its stress-strain curve shows jagged fluctuations, not as smooth as matrix material[26]. On the other hand, different from the failure form of the uniform lattice structure, the other three structures show the form of layer-by-layer destruction. Among them, the gradient lattice structure is gradually destroyed from the top of the entire structure, the other two structures appear to be destroyed layer by layer from bottom to top. Specially, the trend of gradient structure is more obviously damaged by layer-layer with a certain amount of strain ( $\varepsilon=0.2$ ), which is consistent with uniform structure. However, the remaining structures tend to show a failure when the strain was 0.1 , furthermore, the form of destruction becoming clearer until the strain turns 0.2, as shown in Fig.11.
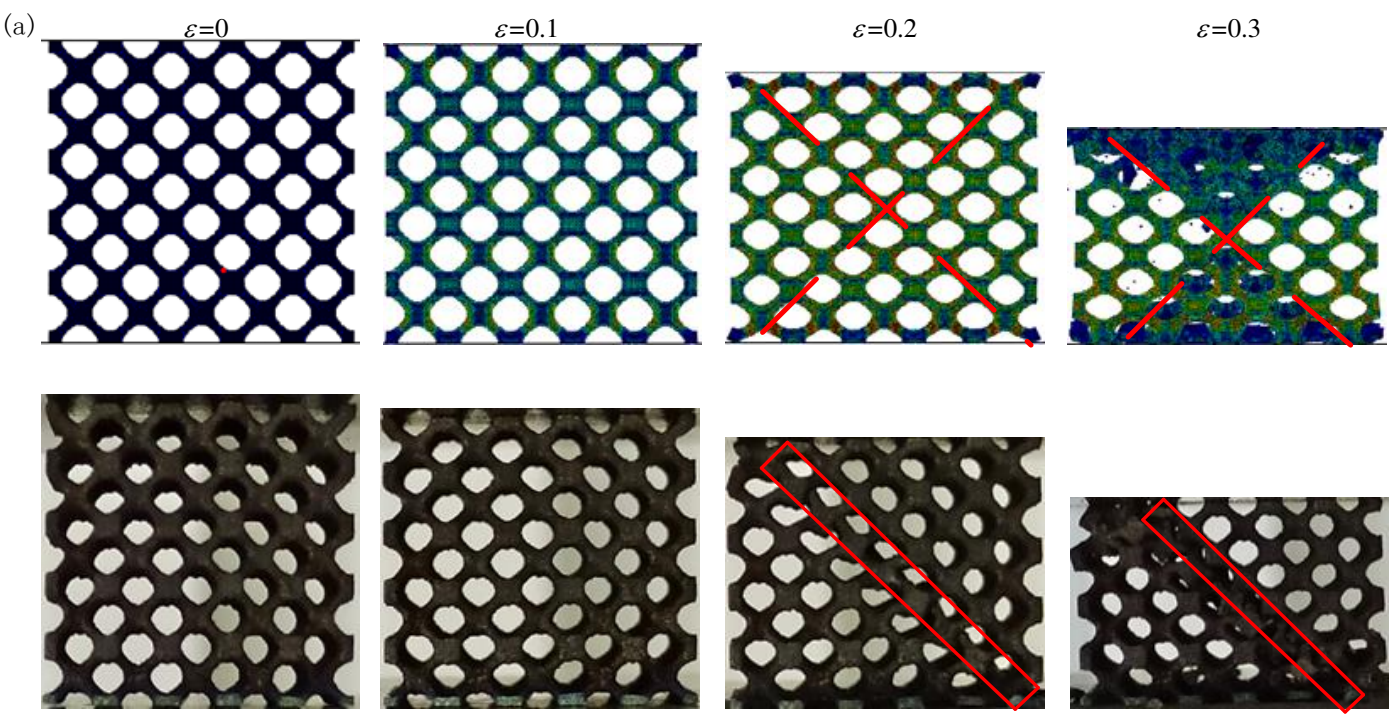

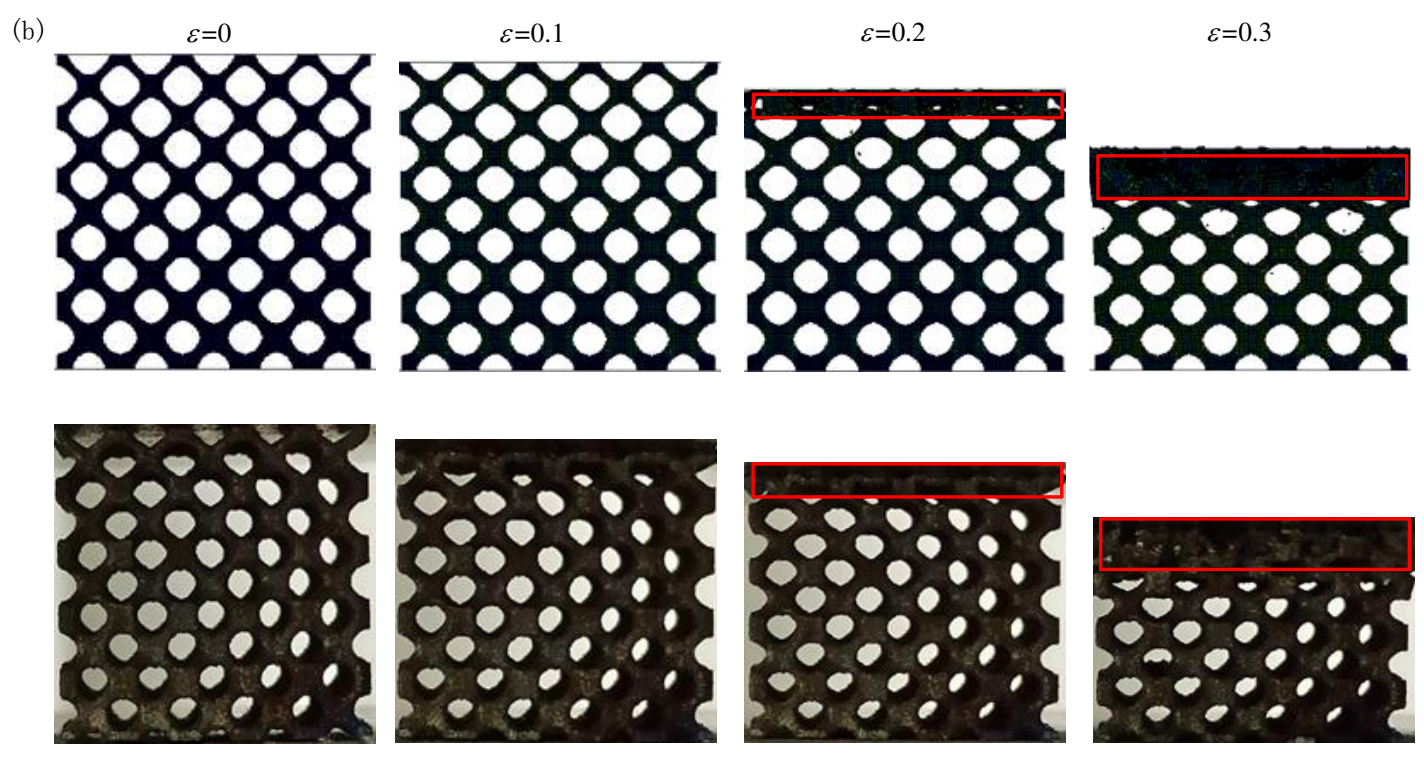

(c)

$\varepsilon=0$

$\varepsilon=0.1$

$\varepsilon=0.2$

$\varepsilon=0.3$
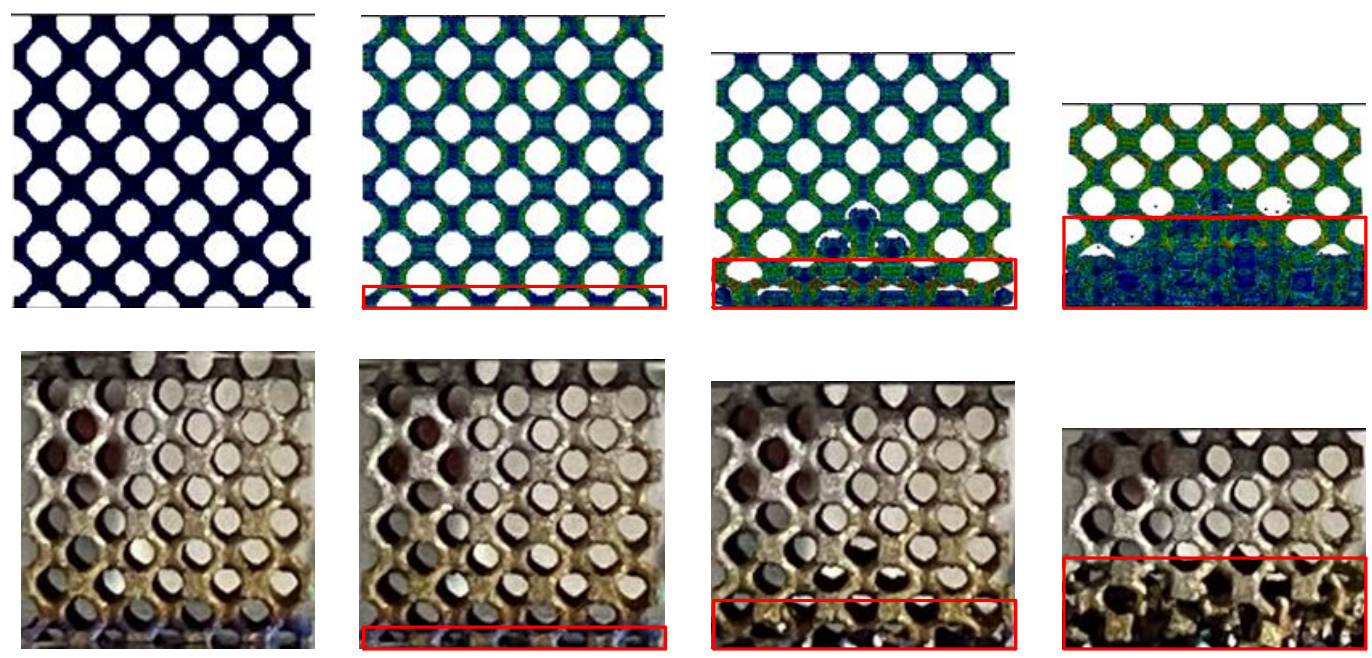

(d)

$\varepsilon=0$

$\varepsilon=0.1$

$\varepsilon=0.2$

$\varepsilon=0.3$
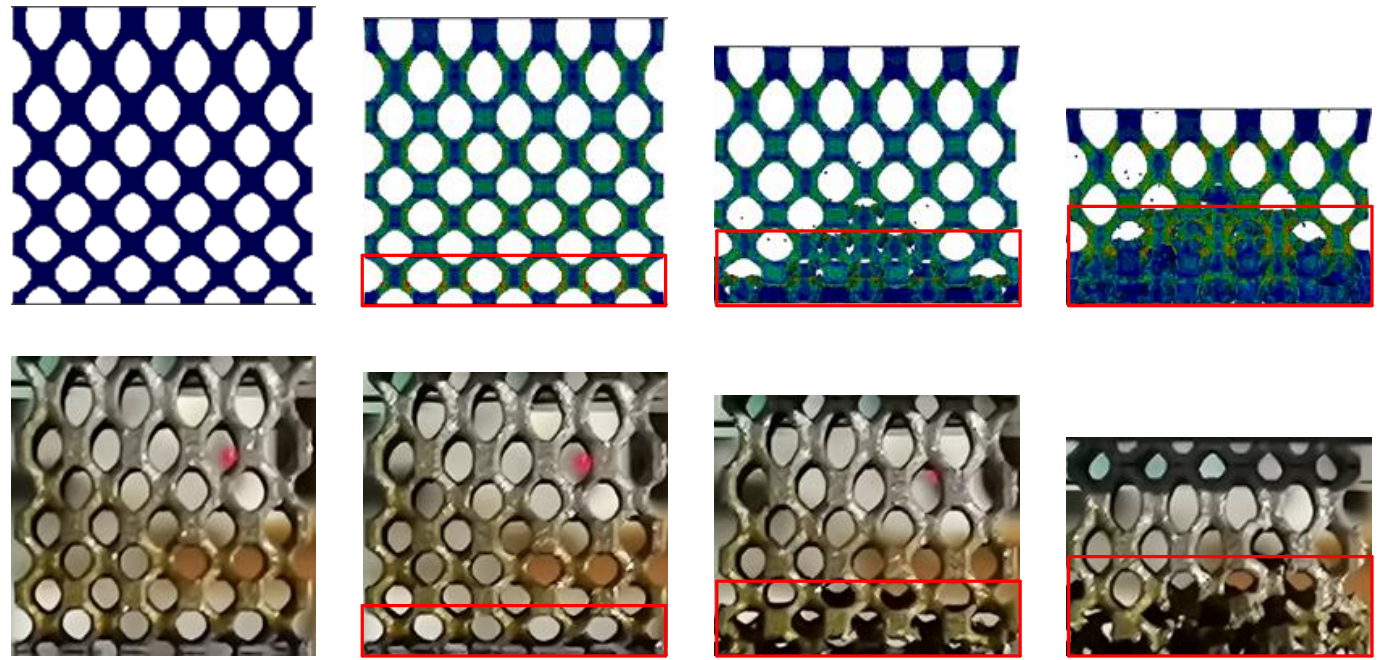

(a) Uniform IW-P; (b) Graded IW-P; (c) LF IW-P;(d) QF IW-P 
Fig.11 Compression deformation process of simulation and experiment

With the compression experiment or simulation, a lot of debris (such as the small black dots in the simulation analysis diagram) were produced by the gradual destruction of the structure, which could further explain the phenomenon of sawtooth-like fluctuations in the stress-strain curve, as shown in Fig.8(a). During the deformation process, the low volume fraction region at the top is destroyed first, and then developed to the surrounding region, while the high-volume fraction area remained not damaged, which could be explained by the stiffness changes in different volume regions, as shown in Fig.8(b). From the screenshots of the simulation analysis, LF IW-P and QF IW-P structures tend obvious tendency of layer-by-layer failure with a certain strain $(\varepsilon=0.1)$, which is a better agreement with the results of the experiment, as shown in Fig.10 and Fig.11.

\subsection{Mechanical properties: modulus and strength}

The elastic modulus and yield strength of the lattice structure are the main indicators to measure the carting capacity of the lattice structure. To evaluate the related mechanical properties of the designed structure, quantitative analysis and comparison of modulus and strength are explored, as shown in Table4. Although the lattice structure researched all based on minimal surface design method, their mechanical properties still show a great difference due to different gradient strategies. As described in Fig.11, the stress-train curves of the four lattice structures are investigated, which show a good agreement with simulation, through compression experiment analysis. Hereinafter, the corresponding mechanical parameters in the experiments of each lattice structure were gained and listed inTable4 by calculating modulus and strength.

It is not difficult to find from Table4 that the elastic modulus and yield strength of the line and second lattice structures are larger, which shows that they have better resistance to external forces and stronger protection under small deformation. Especially, the structure with a linear function gradient in the forming direction is $61.52 \%$ higher than the uniform lattice mechanism under the same volume fraction. Therefore, a lattice structure with linear function gradient change in the building direction may have better mechanical properties by changing the volume fraction, compared with the uniform lattice structures. 
Table4 Comparison of mechanical properties of four lattice structures in experiments

\begin{tabular}{ccccc}
\hline \multirow{2}{*}{ Lattice structure } & \multicolumn{2}{c}{ Elastic modulus (Mpa) } & \multicolumn{2}{c}{ Yield strength (Mpa) } \\
\cline { 2 - 5 } & Sim & Exp & Sim & Exp \\
\hline Uniform Structure & 1002.44 & 1089.48 & 32.42 & 40.72 \\
Graded Structure & 1048.86 & 1028.57 & 23.53 & 28.56 \\
LF Structure & 1350.70 & 1501.65 & 37.71 & 48.37 \\
QF Structure & 1578.24 & 1653.89 & 38.87 & 52.41 \\
\hline
\end{tabular}

3.4 Energy absorption under compression deformation

Due to its high porosity, lattice structure has the characteristics of lightweight and energy absorption. Therefore, it is always used in protective devices since energy would be absorbed during deformation. According to ISO 13314:2011, the energy absorption of those lattice structure was evaluated. The calculation formula of energy absorption is expressed as follows.

$$
W=\int_{0}^{\varepsilon_{d}} \sigma(\varepsilon) d \varepsilon
$$

Herein, $\mathrm{W}$ is the energy absorption per lattice unit volume which is defined as the area enclosed by the stress-strain curves and $\mathrm{x}$-axis, $\sigma$ and $\varepsilon$ represent compression stress and strain, respectively, and $\varepsilon_{d}$ is the maximum strain at the onset of the densification stage. The results are outlined in Table5. It can be found that the graded lattice structure absorbed a higher amount of energy as compared to the uniform lattice with the same volume fraction(20\%), which agrees with the results from the literature [30]. Hereinafter, only $50 \%$ of the calculated value is selected for energy absorption, which is consistent with the calculation methods of other documents[30]. By integrating each strain position, the energy absorption curve of the lattice structures under different strains was acquired, as shown in Fig.12. 


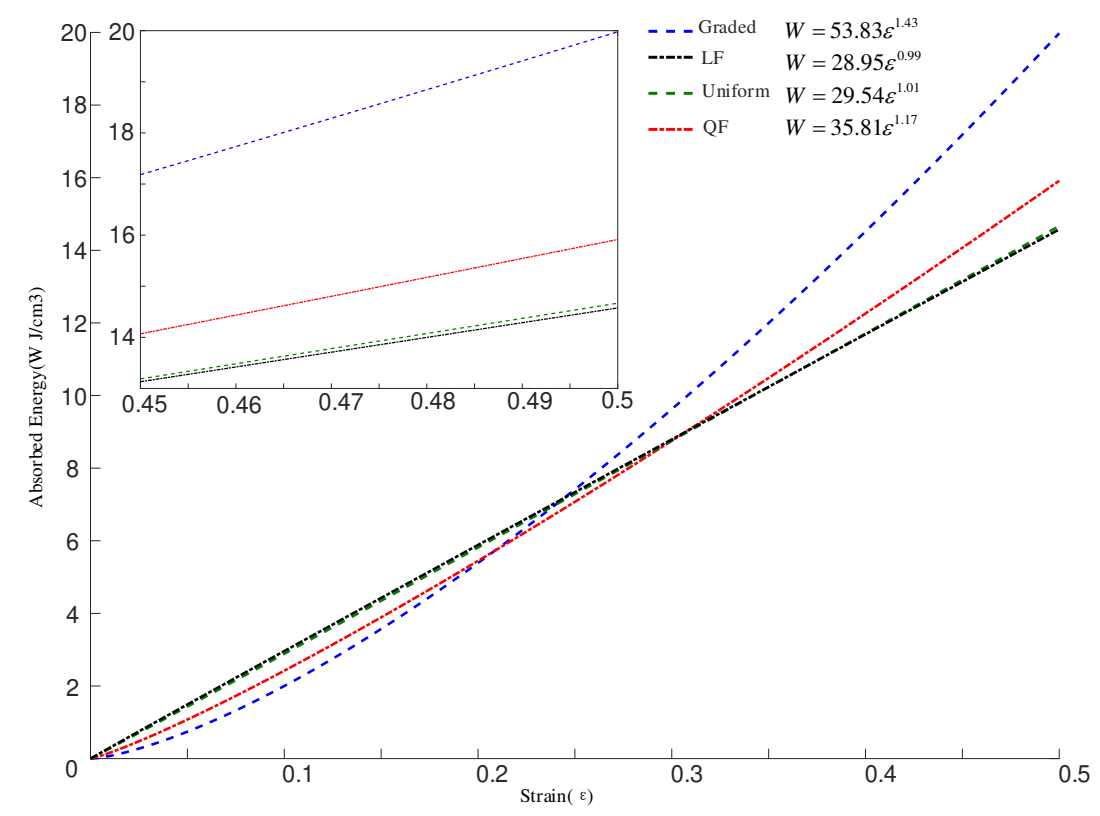

Fig.12 Energy absorption curves of the lattice structures

It can be clearly seen from Fig. 12 that the LF lattice structure has an energy absorption capacity equivalent to that of the uniform lattice structure. Compared with the other lattice structures, however, the gradient lattice structure has the lowest energy absorption capacity during a certain strain $(\varepsilon=0-0.25)$. During the second half of the compression experiment, the graded lattice structure performs more strongly energy absorption capacity $(\varepsilon=0.25-0.5)$. On the other hand, the QF lattice structure has the ability of energy absorbed preceded only by the graded lattice at the stage where the strain during a certain strain $(\varepsilon=0.3-0.5)$.

Table5 Energy absorption property of lattice samples

\begin{tabular}{cccccccccc}
\hline \multirow{2}{*}{ Properties } & \multicolumn{2}{c}{ Uniform } & \multicolumn{2}{c}{ Graded } & \multicolumn{2}{c}{ LF } & \multicolumn{2}{c}{ QF } \\
\cline { 2 - 10 } & Exp & Sim & Exp & Sim & Exp & Sim & Exp & Sim \\
\hline W(MJ/m3) & 14.67 & 13.72 & 20.34 & 18.45 & 13.49 & 14.55 & 16.39 & 15.74 \\
\hline
\end{tabular}

As shown in Table5, the errors of energy absorption for the lattice structures (uniform lattice, gradient lattice, LF and QF) during simulation and compression experiment are 7.28\%, 10.08\%, $1.82 \%$ and $3.75 \%$ respectively. Specifically, the energy absorption of the QF lattice structure is $10.94 \%$ higher than that of the uniform lattice structure in the compression experiment, and the simulation is $14.72 \%$.

Furthermore, the lattice structure with the linear function gradient strategy in the manufacturing direction has the energy absorption capacity of the strain power function, which shows consistent 
performance with the uniform lattice structure. Nevertheless, the lattice structure adopting the quadratic function gradient strategy in the forming direction has almost the same energy absorption capacity as the lattice structure applying the gradual volume fraction in the fabricating direction, which provides a variety of design solutions for engineering and technical person in the field of lightweight applications.

\section{Conclusion}

In this work, the uniform, graded, LF and QF IW-P lattice structures based on TPMS were fabricated via SLM with TC4 power, with the same volume fraction $20 \%$ and unit cell size of $4 \mathrm{~mm}$. Furthermore, their mechanical properties, compression deformation behaviors and energy absorption properties have been experimentally and numerically investigated. The main conclusions are as follows.

(1) Compared with uniform and graded IW-P lattice structures, LF and QF IW-P lattice structures showed higher elastic modulus and yield strength with the same volume fraction. The deformation behavior of the latter three lattice structures occurred a brittle layer-by-layer collapse, nevertheless, the uniform IW-P lattice structure was destroyed in the range of direction of $45^{\circ}$.

(2) The plastic deformation and layer-by-layer failure of these four lattice structures were simulated with the introduced Johnson-Cook and damage models, and the deformation mechanism was consistent with experimental results. Hereafter, the method of predicting the mechanical properties (especially in yield strength, compressive strength and energy absorption) of the lattice structure is a constructive guide for the structural designer.

(3) In view of the total absorption, uniform and QF lattice structure have a higher energy absorption ability than graded and line lattice structures. Specifically, the energy absorption of former structures increased along with the power function of strain, whereas the latter ones occurred almost linear growth. This phenomenon illustrates the effectiveness and rationality of the gradient design method proposed in the article.

In conclusion, QF IW-P ones have higher Elastic modulus and yield strength with the same volume fraction. In terms of energy absorption, the uniform IW-P and QF IW-P lattice structures show better potential. The mechanicals behaviors of lattice structures based on TPMS could be predicted accurately by the FEA. However, this study only focuses on the design method of lattice 
structures and the mechanical properties. With the demand for lightweight in the manufacturing, aerospace and automotive fields, the basic principles behind the design of the lattice structure, the design method of the lattice structure and its lightweight application will be the focus of future research

\section{Declaration}

Funding: This work was supported by Key projects of Chongqing Natural Science Foundation [cstc2020jcyj-zdxmX0021], National High Technology Research and Development Program of China (863Program:2015AA042501).

Conflicts of interest/Competing interests: The authors declare that they have no competing interests. Availability of data and material: Not applicable

Code availability: Not applicable

Ethics approval: Not applicable

Consent to participate: Not applicable

Consent for publication: Not applicable

Authors' contributions: Xiangyu Ma and David Z. Zhang designed the work, performed the research, and analyzed the data. Xiangyu Ma, David Z. Zhang, Miao Zhao, Junjie Jiang, Fangqiong Luo and Hailun Zhou discussed the results and wrote the manuscript. All authors contributed to drafting and revising the manuscript.

\section{Reference}

[1] Z. Liu, Y. Zhang, M. Zhang, G. Tan, Y. Zhu, Z. Zhang, R.O. Ritchie, Adaptive structural reorientation: Developing extraordinary mechanical properties by constrained flexibility in natural materials, Acta Biomaterialia, 86 (2019) 96-108.

[2] L. Ren, B. Li, Z. Song, Q. Liu, L. Ren, X. Zhou, 3D printing of structural gradient soft actuators by variation of bioinspired architectures, Journal of Materials Science, 54 (2019) 6542-6551.

[3] G. Chen, H. Luo, Z. Zhang, X. Fan, Flexural deformation and fracture behaviors of the sandwich turtle rib bones with hierarchical woven fibers, Colloid and Interface Science Communications, 34 (2020).

[4] J. Ott, M. Lazalde, G.X. Gu, Algorithmic-driven design of shark denticle bioinspired structures for superior aerodynamic properties, Bioinspiration \& Biomimetics, 15 (2020).

[5] S. Mannan, J. Paul Knox, S. Basu, Correlations between axial stiffness and microstructure of a species of bamboo, R Soc Open Sci, 4 (2017) 160412.

[6] M. Frey, G. Biffi, M. Adobes-Vidal, M. Zirkelbach, Y. Wang, K. Tu, A.M. Hirt, K. Masania, I. Burgert, T. Keplinger, Tunable Wood by Reversible Interlocking and Bioinspired Mechanical 
Gradients, Advanced Science, 6 (2019).

[7] W. Luo, H. Liu, C. Wang, Y. Qin, Q. Liu, J. Wang, Bioprinting of Human Musculoskeletal Interface, Advanced Engineering Materials, 21 (2019).

[8] D. Chen, Q. Liu, Z. Han, J. Zhang, H. Song, K. Wang, Z. Song, S. Wen, Y. Zhou, C. Yan, Y. Shi, 4D Printing Strain Self-Sensing and Temperature Self-Sensing Integrated Sensor-Actuator with Bioinspired Gradient Gaps, Advanced Science, 7 (2020).

[9] Y. Jin, H.Y. Kong, X.Y. Zhou, G.Y. Li, J.K. Du, Design and Characterization of Sheet-Based Gyroid Porous Structures with Bioinspired Functional Gradients, Materials, 13 (2020).

[10] S. Das, A. Sutradhar, Multi-physics topology optimization of functionally graded controllable porous structures: Application to heat dissipating problems, Materials \& Design, 193 (2020) 108775. [11] I. Kaur, P. Singh, Flow and thermal transport characteristics of Triply-Periodic Minimal Surface (TPMS)-based gyroid and Schwarz-P cellular materials, Numerical Heat Transfer Part a-Applications.

[12] Y.J. Liang, W. Zhou, Y.J. Liu, Z.S. Li, Y. Yang, H.F. Xi, Z.G. Wu, Energy Absorption and Deformation Behavior of 3D Printed Triply Periodic Minimal Surface Stainless Steel Cellular Structures under Compression, Steel Research International.

[13] R. Alberdi, R. Dingreville, J. Robbins, T. Walsh, B.C. White, B. Jared, B.L. Boyce, Multimorphology lattices lead to improved plastic energy absorption, Materials \& Design, 194 (2020). [14] J. Plocher, A. Panesar, Effect of density and unit cell size grading on the stiffness and energy absorption of short fibre-reinforced functionally graded lattice structures, Additive Manufacturing, 33 (2020).

[15] L.J. Xiao, X. Xu, W.D. Song, M.L. Hu, A Multi-Cell Hybrid Approach to Elevate the Energy Absorption of Micro-Lattice Materials, Materials, 13 (2020).

[16] I. Bharti, N. Gupta, K.M. Gupta, Novel Applications of Functionally Graded Nano, Optoelectronic and Thermoelectric Materials, International Journal of Materials, Mechanics and Manufacturing, (2013) 221-224.

[17] A. Ataee, Y. Li, M. Brandt, C. Wen, Ultrahigh-strength titanium gyroid scaffolds manufactured by selective laser melting (SLM) for bone implant applications, Acta Materialia, 158 (2018) 354-368.

[18] W. Xu, X. Lu, M.D. Hayat, J. Tian, C. Huang, M. Chen, X. Qu, C. Wen, Fabrication and properties of newly developed Ti35Zr28Nb scaffolds fabricated by powder metallurgy for bone-tissue engineering, Journal of Materials Research and Technology, 8 (2019) 3696-3704.

[19] W. Xu, J. Tian, Z. Liu, X. Lu, M.D. Hayat, Y. Yan, Z. Li, X. Qu, C. Wen, Novel porous

Ti35Zr28Nb scaffolds fabricated by powder metallurgy with excellent osteointegration ability for bonetissue engineering applications, Mater Sci Eng C Mater Biol Appl, 105 (2019) 110015.

[20] S. Vijayavenkataraman, L.Y. Kuan, W.F. Lu, 3D-printed ceramic triply periodic minimal surface structures for design of functionally graded bone implants, Materials \& Design, 191 (2020) 108602.

[21] R. Xiao, X. Feng, R. Fan, S. Chen, J. Song, L. Gao, Y. Lu, 3D printing of titanium-coated gradient composite lattices for lightweight mandibular prosthesis, Composites Part B: Engineering, 193 (2020) 108057.

[22] Z. Jun, A. Xing, D. Jianxin, W. Jinghai, Thermal shock behaviors of functionally graded ceramic tool materials, Journal of the European Ceramic Society, 24 (2004) 847-854.

[23] I. Konyashin, A.A. Zaitsev, D. Sidorenko, E.A. Levashov, S.N. Konischev, M. Sorokin, S. Hlawatschek, B. Ries, A.A. Mazilkin, S. Lauterbach, H.J. Kleebe, On the mechanism of obtaining functionally graded hardmetals, Materials Letters, 186 (2017) 142-145.

[24] M.Z. Nejad, N. Alamzadeh, A. Hadi, Thermoelastoplastic analysis of FGM rotating thick 
cylindrical pressure vessels in linear elastic-fully plastic condition, Composites Part B: Engineering, 154 (2018) 410-422.

[25] B. Saleh, J. Jiang, R. Fathi, T. Al-hababi, Q. Xu, L. Wang, D. Song, A. Ma, 30 Years of functionally graded materials: An overview of manufacturing methods, Applications and Future Challenges, Composites Part B: Engineering, 201 (2020) 108376.

[26] L. Bai, C. Gong, X. Chen, Y. Sun, L. Xin, H. Pu, Y. Peng, J. Luo, Mechanical properties and energy absorption capabilities of functionally graded lattice structures: Experiments and simulations, International Journal of Mechanical Sciences, 182 (2020) 105735.

[27] M.J. Mirzaali, A.H. de la Nava, D. Gunashekar, M. Nouri-Goushki, R.P.E. Veeger, Q. Grossman, L. Angeloni, M.K. Ghatkesar, L.E. Fratila-Apachitei, D. Ruffoni, E.L. Doubrovski, A.A. Zadpoor, Mechanics of bioinspired functionally graded soft-hard composites made by multi-material 3D printing, Composite Structures, 237 (2020).

[28] F. Liu, Z. Mao, P. Zhang, D.Z. Zhang, J. Jiang, Z. Ma, Functionally graded porous scaffolds in multiple patterns: New design method, physical and mechanical properties, Materials \& Design, 160 (2018) 849-860.

[29] Z. Ma, D.Z. Zhang, F. Liu, J. Jiang, M. Zhao, T. Zhang, Lattice structures of Cu-Cr-Zr copper alloy by selective laser melting: Microstructures, mechanical properties and energy absorption, Materials \& Design, 187 (2020) 108406.

[30] H. Zhou, M. Zhao, Z. Ma, D.Z. Zhang, G. Fu, Sheet and network based functionally graded lattice structures manufactured by selective laser melting: Design, mechanical properties, and simulation, International Journal of Mechanical Sciences, 175 (2020) 105480.

[31] O. Al-Ketan, D.W. Lee, R. Rowshan, R.K. Abu Al-Rub, Functionally graded and multimorphology sheet TPMS lattices: Design, manufacturing, and mechanical properties, J Mech Behav Biomed Mater, 102 (2020) 103520.

[32] L. Yang, C. Yan, W. Cao, Z. Liu, B. Song, S. Wen, C. Zhang, Y. Shi, S. Yang, Compressioncompression fatigue behaviour of gyroid-type triply periodic minimal surface porous structures fabricated by selective laser melting, Acta Materialia, 181 (2019) 49-66.

[33] H. Jia, H. Lei, P. Wang, J. Meng, C. Li, H. Zhou, X. Zhang, D. Fang, An experimental and numerical investigation of compressive response of designed Schwarz Primitive triply periodic minimal surface with non-uniform shell thickness, Extreme Mechanics Letters, 37 (2020).

[34] D.A. de Aquino, I. Maskery, G.A. Longhitano, A.L. Jardini, E.G. del Conte, Investigation of load direction on the compressive strength of additively manufactured triply periodic minimal surface scaffolds, International Journal of Advanced Manufacturing Technology, 109 (2020) 771-779.

[35] I. Maskery, I.A. Ashcroft, The deformation and elastic anisotropy of a new gyroid-based honeycomb made by laser sintering, Additive Manufacturing, 36 (2020).

[36] F. Liu, Q. Ran, M. Zhao, T. Zhang, D.Z. Zhang, Z. Su, Additively Manufactured Continuous CellSize Gradient Porous Scaffolds: Pore Characteristics, Mechanical Properties and Biological Responses In Vitro, Materials (Basel), 13 (2020).

[37] M. Zhao, D.Z. Zhang, F. Liu, Z. Li, Z. Ma, Z. Ren, Mechanical and energy absorption characteristics of additively manufactured functionally graded sheet lattice structures with minimal surfaces, International Journal of Mechanical Sciences, 167 (2020) 105262.

[38] X. Zhang, J. Wang, T. Liu, 3D printing of polycaprolactone-based composites with diversely tunable mechanical gradients via multi-material fused deposition modeling, Composites Communications, 23 (2021) 100600. 
[39] H. Zhou, M. Zhao, Z. Ma, D.Z. Zhang, G. Fu, Sheet and network based functionally graded lattice structures manufactured by selective laser melting: Design, mechanical properties, and simulation, International Journal of Mechanical Sciences, 175 (2020).

[40] A.V. Sobolev, M.V. Radchenko, Use of Johnson-Cook plasticity model for numerical simulations of the SNF shipping cask drop tests, Nuclear Energy and Technology, 2 (2016) 272-276.

[41] M. Zhao, D.Z. Zhang, F. Liu, Z. Li, Z. Ma, Z. Ren, Mechanical and energy absorption characteristics of additively manufactured functionally graded sheet lattice structures with minimal surfaces, International Journal of Mechanical Sciences, 167 (2020).

[42] Y. Liang, W. Zhou, Y. Liu, Z. Li, Y. Yang, H. Xi, Z. Wu, Energy Absorption and Deformation Behavior of 3D Printed Triply Periodic Minimal Surface Stainless Steel Cellular Structures under Compression, Steel Research International, (2020).

[43] L. Yang, M. Ferrucci, R. Mertens, W. Dewulf, C. Yan, Y. Shi, S. Yang, An investigation into the effect of gradients on the manufacturing fidelity of triply periodic minimal surface structures with graded density fabricated by selective laser melting, Journal of Materials Processing Technology, 275 (2020) 116367.

[44] F. Calignano, M. Lorusso, J. Pakkanen, F. Trevisan, E.P. Ambrosio, D. Manfredi, P. Fino, Investigation of accuracy and dimensional limits of part produced in aluminum alloy by selective laser melting, The International Journal of Advanced Manufacturing Technology, 88 (2016) 451-458.

[45] C. Qiu, S. Yue, N.J.E. Adkins, M. Ward, H. Hassanin, P.D. Lee, P.J. Withers, M.M. Attallah, Influence of processing conditions on strut structure and compressive properties of cellular lattice structures fabricated by selective laser melting, Materials Science and Engineering: A, 628 (2015) 188197.

[46] X. Guo, X. Zheng, Y. Yang, X. Yang, Y. Yi, Mechanical behavior of TPMS-based scaffolds: a comparison between minimal surfaces and their lattice structures, Sn Applied Sciences, 1 (2019). [47] Z. Wang, X. Wang, T. Gao, C. Shi, Mechanical behavior and deformation mechanism of triply periodic minimal surface sheet under compressive loading, Mechanics of Advanced Materials and Structures, (2020). 


\section{Figures}

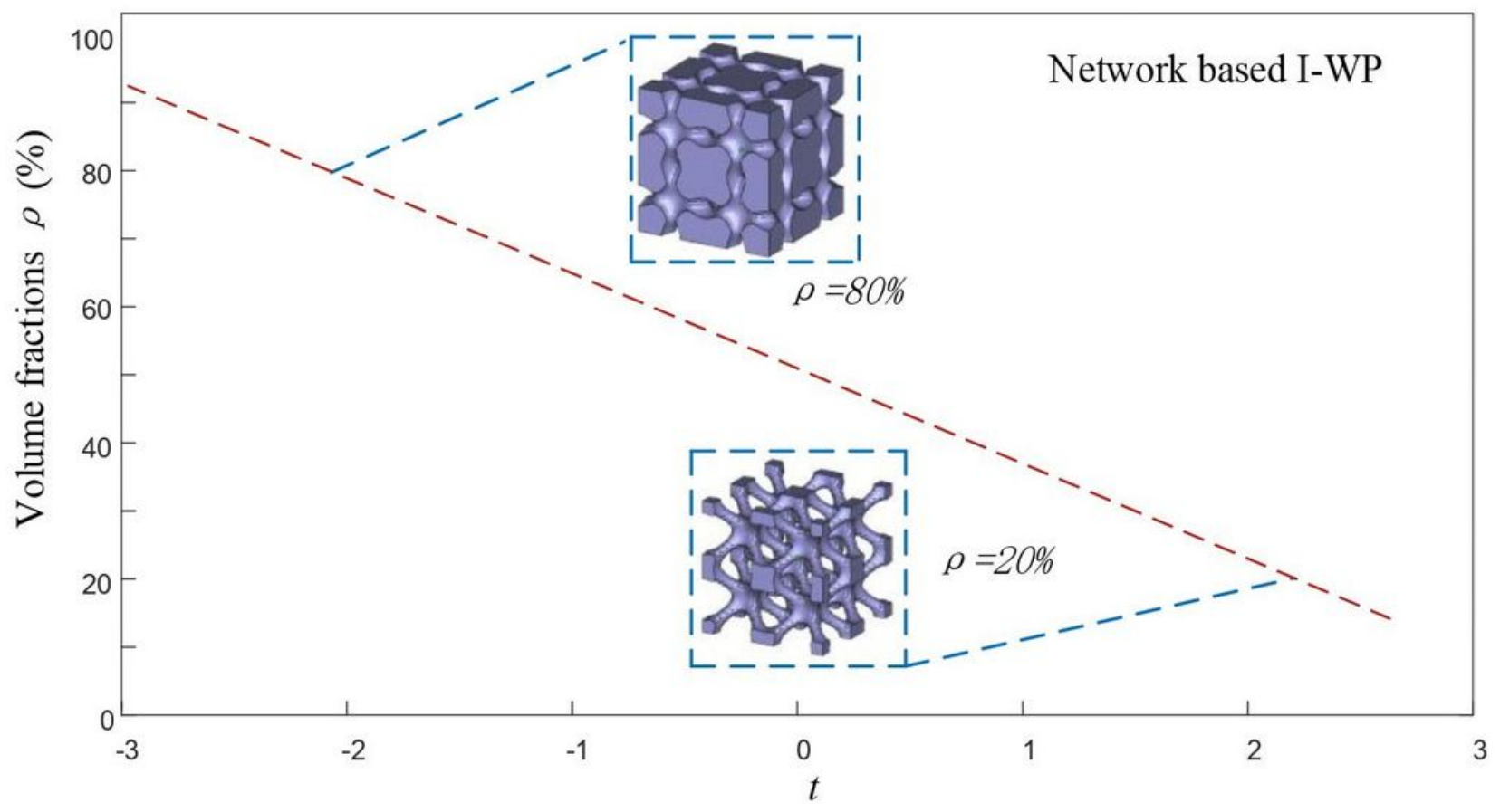

Figure 1

The functional relationship between $t$ and $\rho$ of network based I-WP structures.
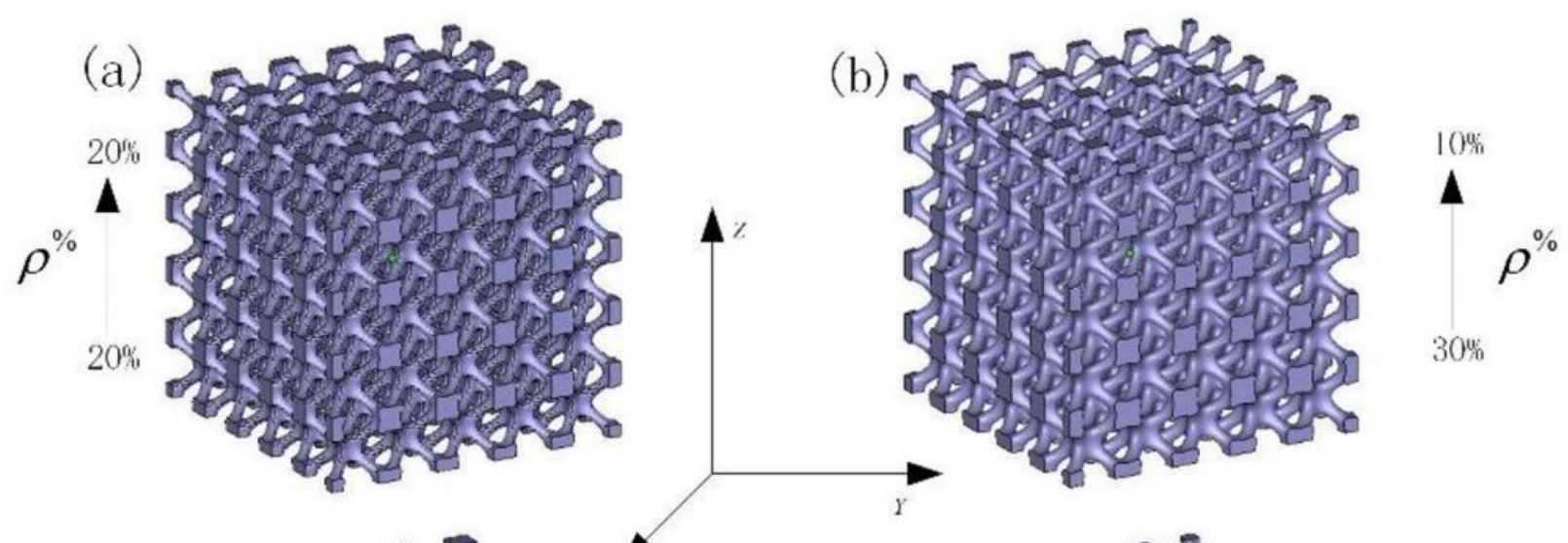

(c)

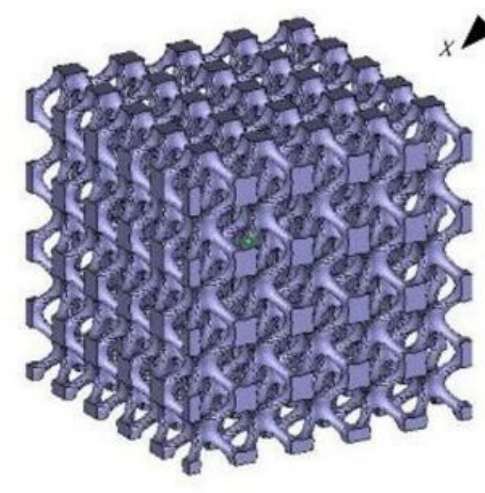

(d)

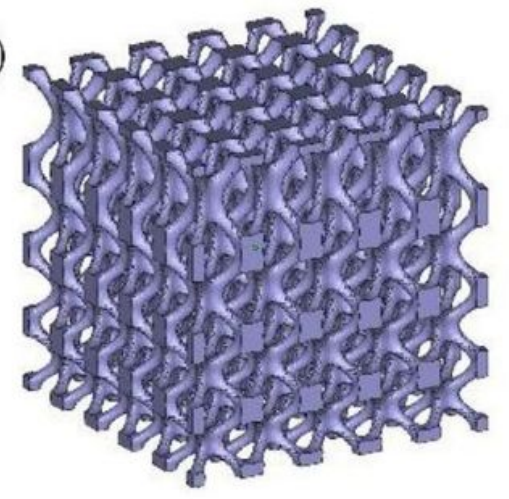


Figure 2

Three-dimensional models of (a) Uniform IW-P; (b)Graded IW-P; (c) LF IW-P and (d) QF IW-P lattice structures
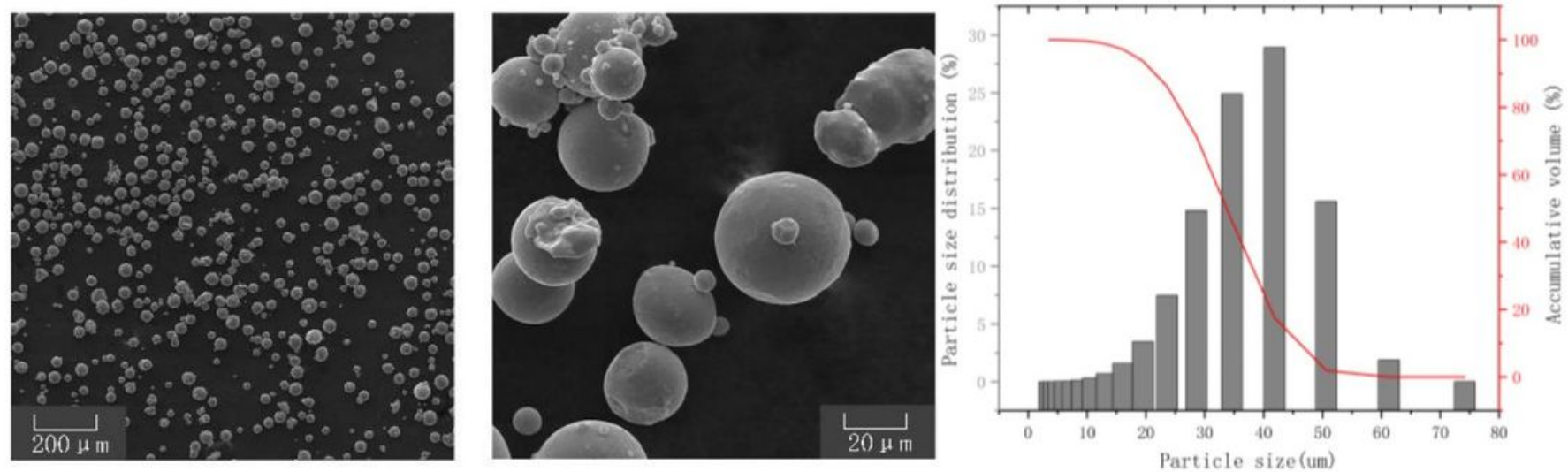

(a) SEM micrograph

(b) particle size distribution

Figure 3

(a) SEM micrograph and (b) particle size distribution of TC4 powder
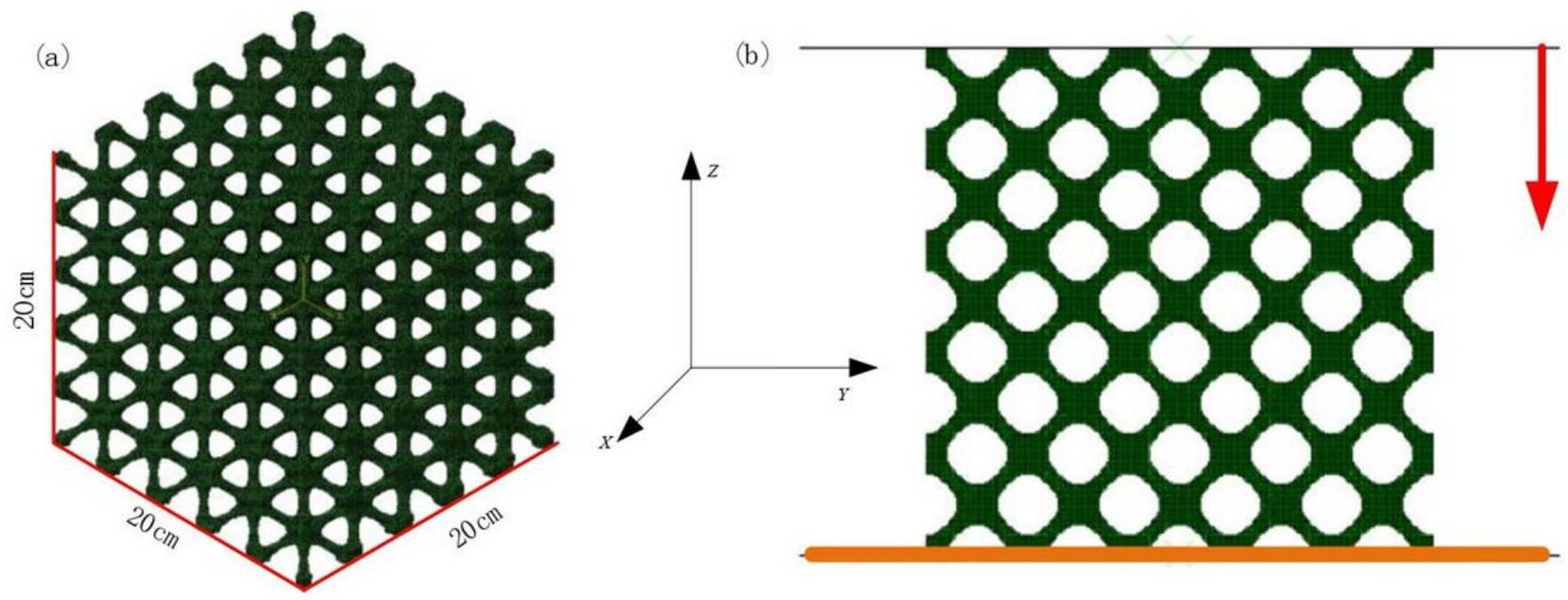

Figure 4

The FE models of hexahedral meshes: (a) Uniform IW-P (with 20\% volume fraction) based FE model; (b) the boundary condition in FEA 


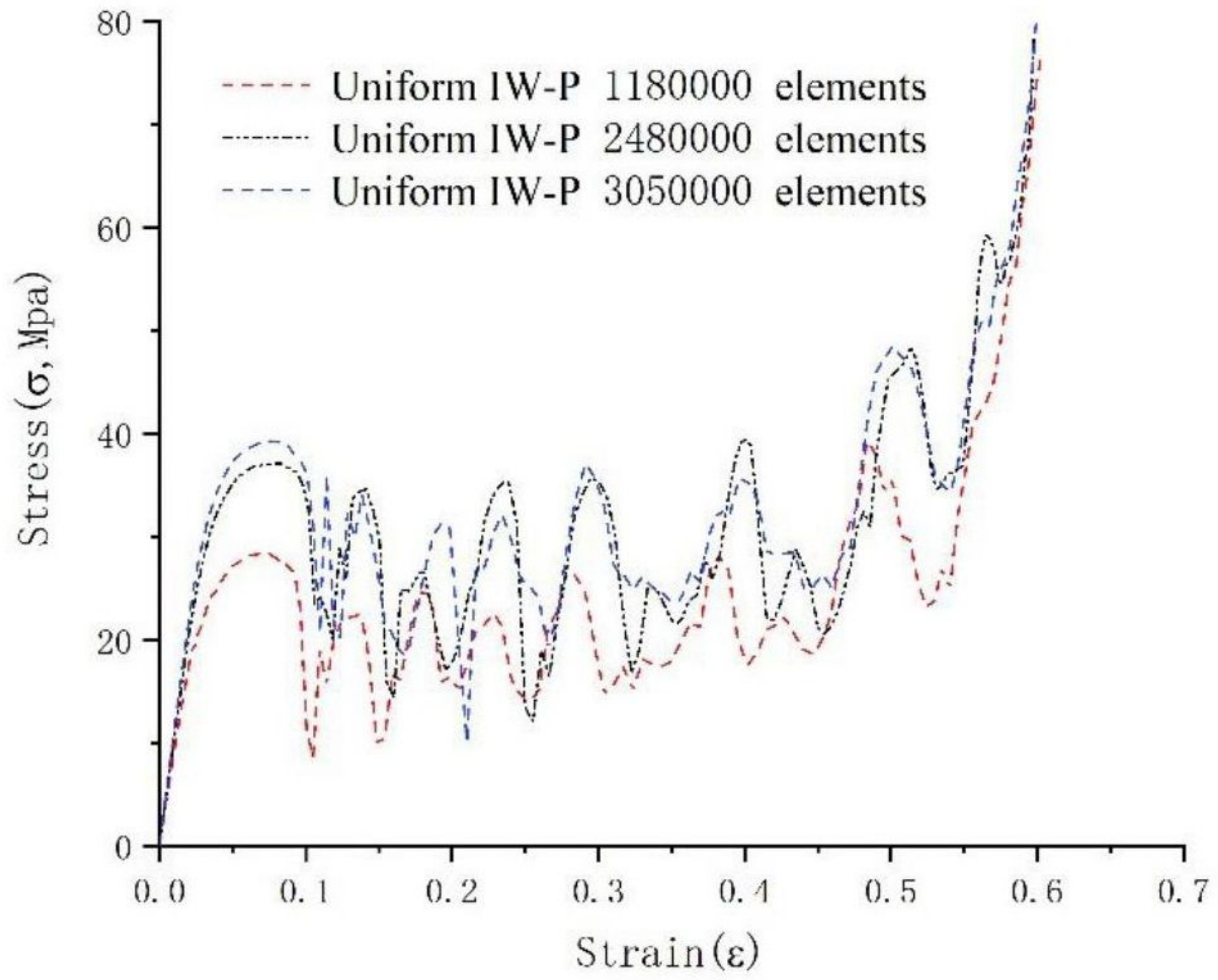

Figure 5

Convergence of stress-strain curves corresponding to the three mesh numbers for the W20 structure.

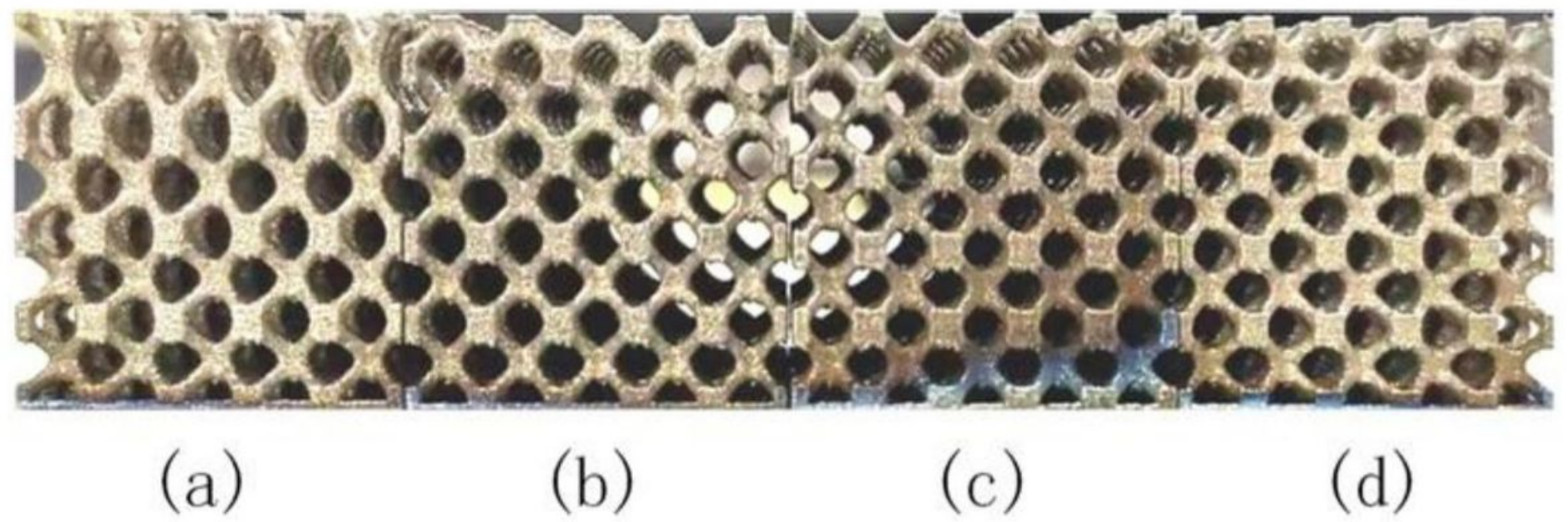


Figure 6

(a)QF IW-P; (b) LF IW-P; (c) Graded IW-P; (d) Uniform IW-P

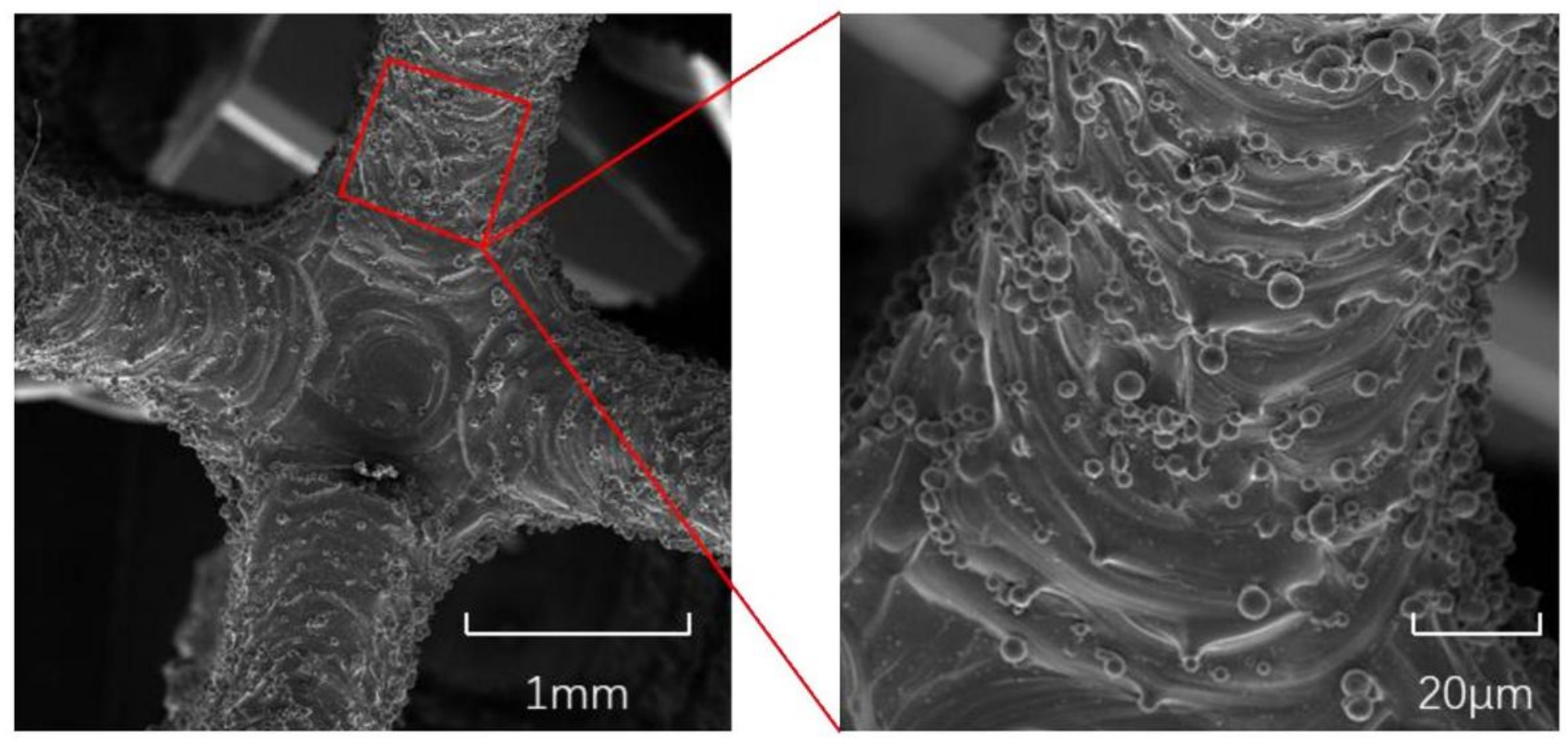

Figure 7

SEM morphology of uniform IW-P structure's surface 


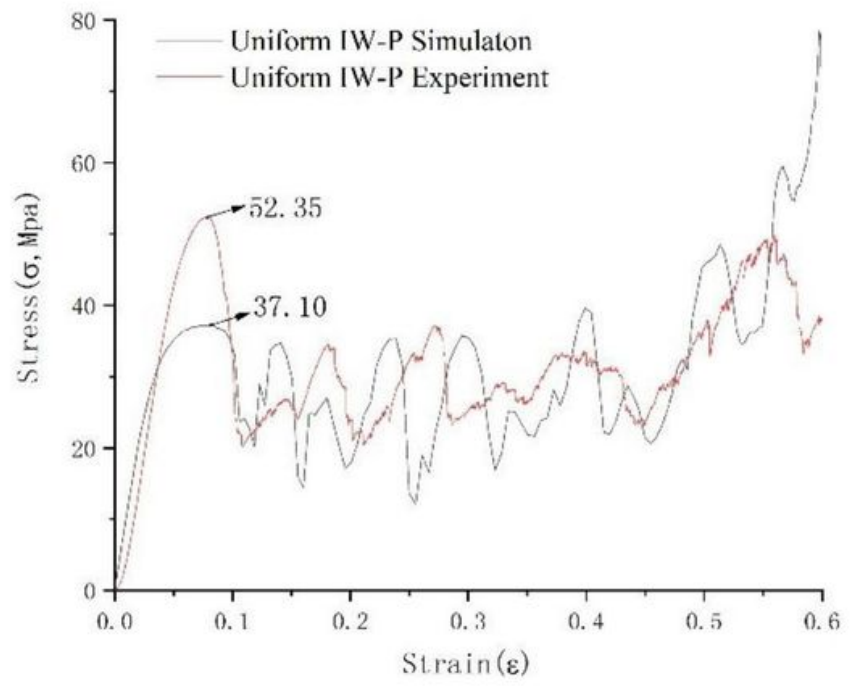

(a) Uniform IW-P

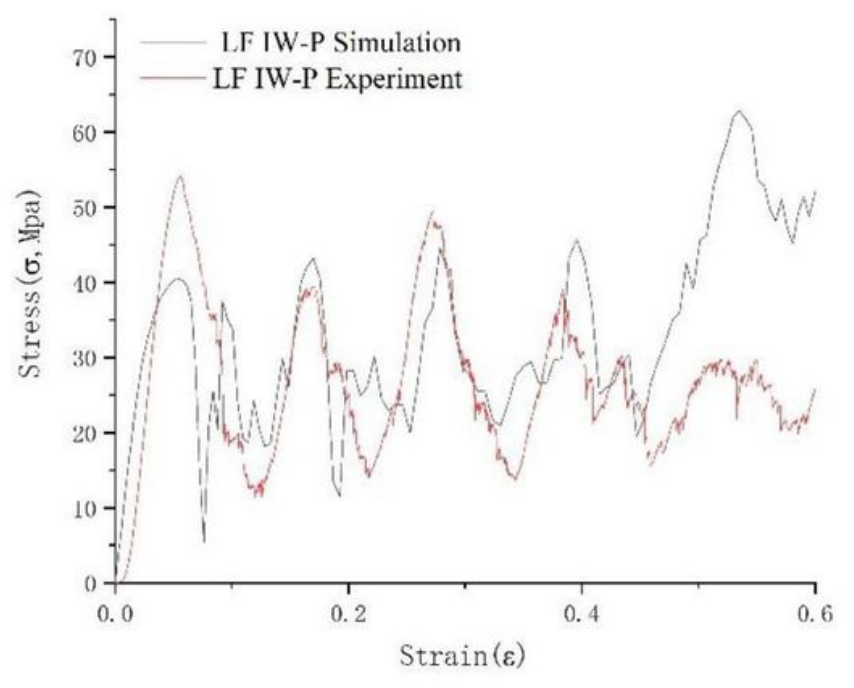

(c) LF IW-P

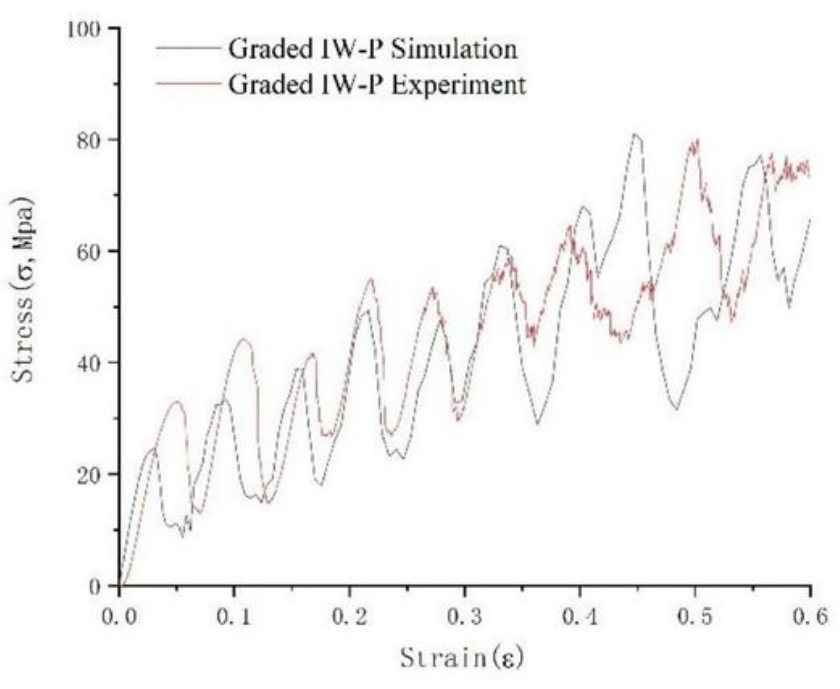

(b) Graded IW-P

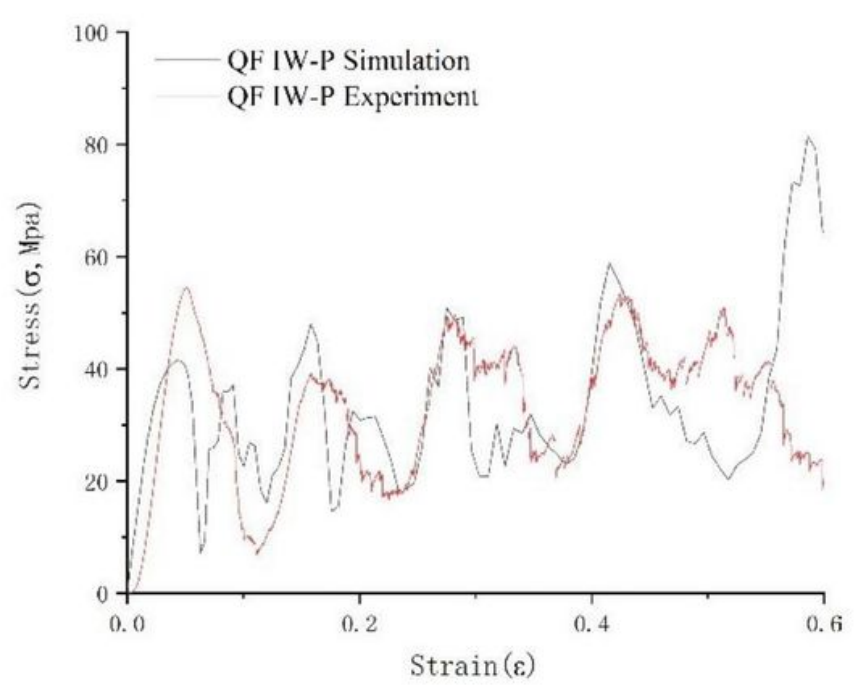

(d) QF IW-P

\section{Figure 8}

Simulation and experimental curve comparison of four lattice structures 


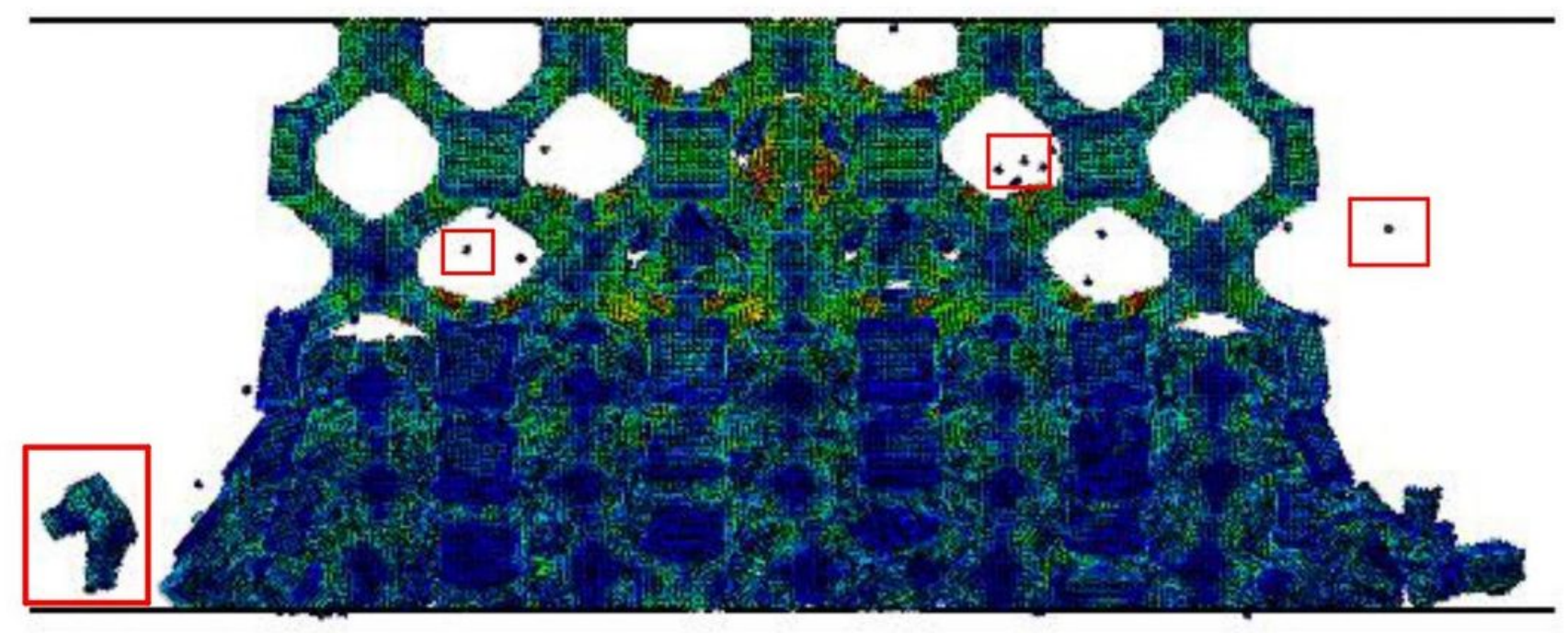

Figure 9

Schematic diagram of LF IW-P lattice structure simulation analysis fragments

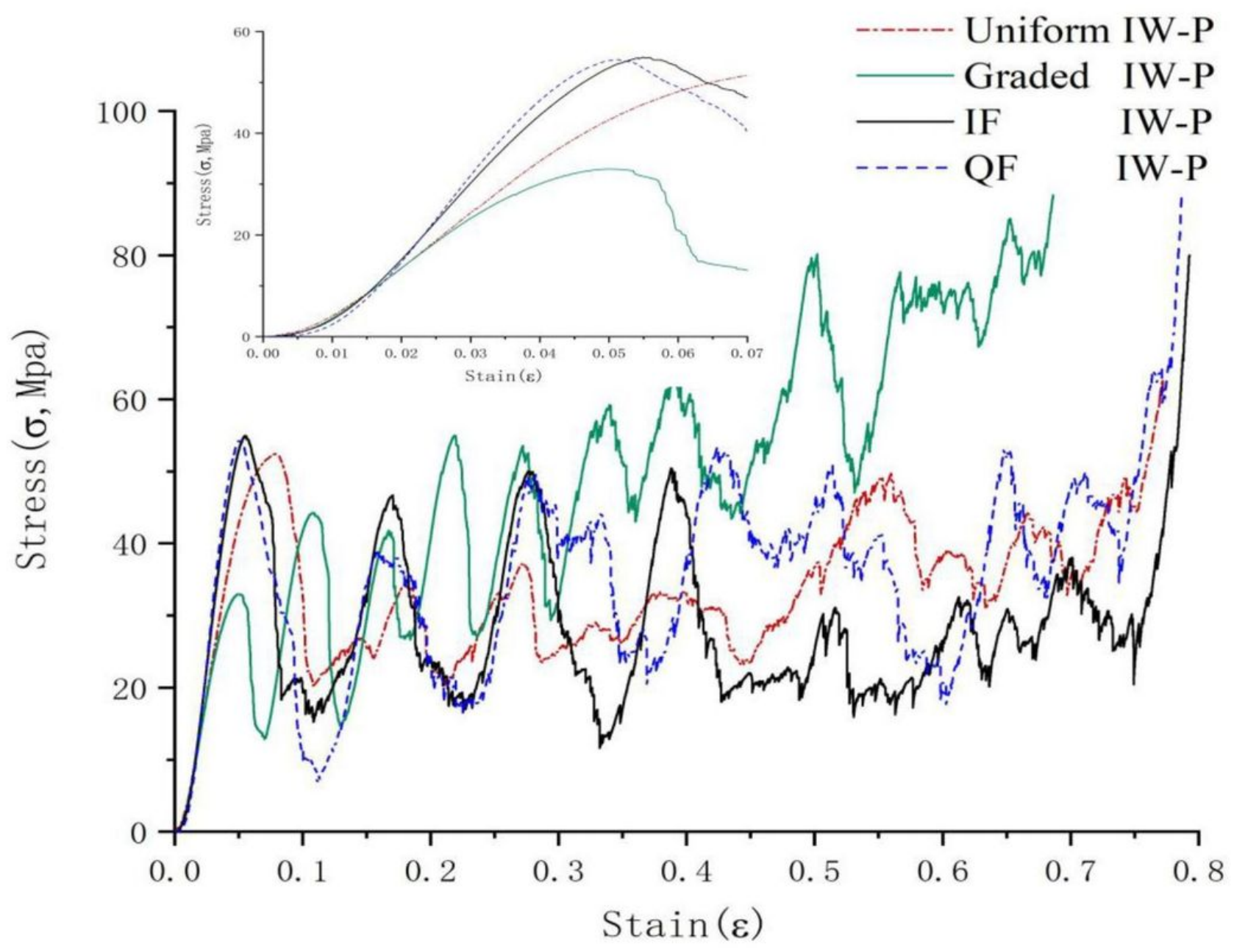


Figure 10

The comparison with experimental results of lattice structure
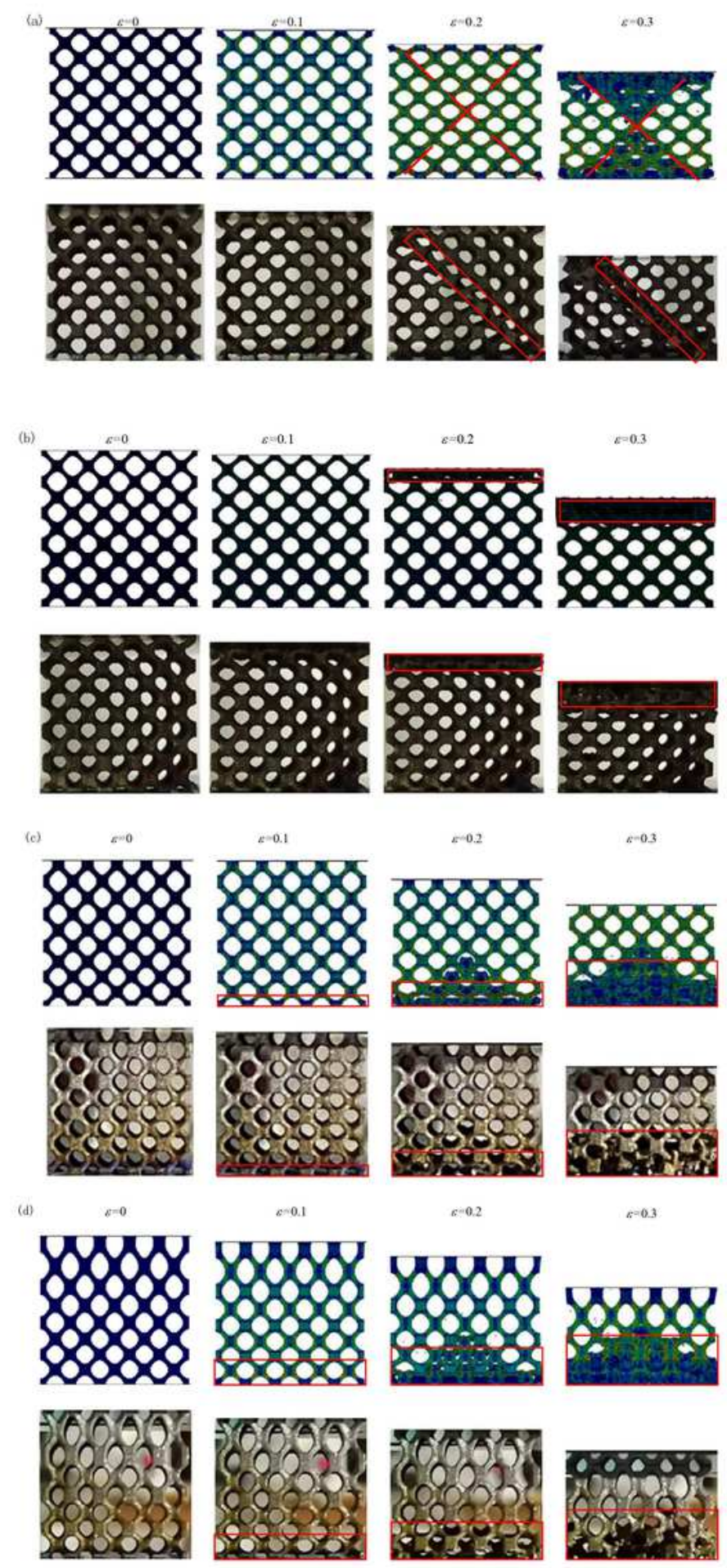

$\because 0.1$

${ }^{6}=0.2$

${ }^{2}=0.3$
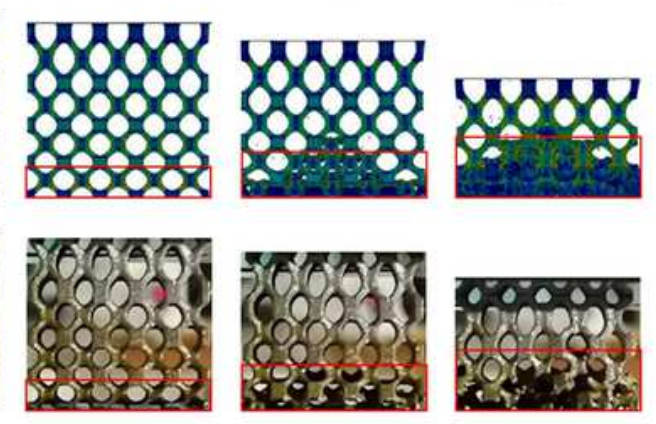

Figure 11

Compression deformation process of simulation and experiment 


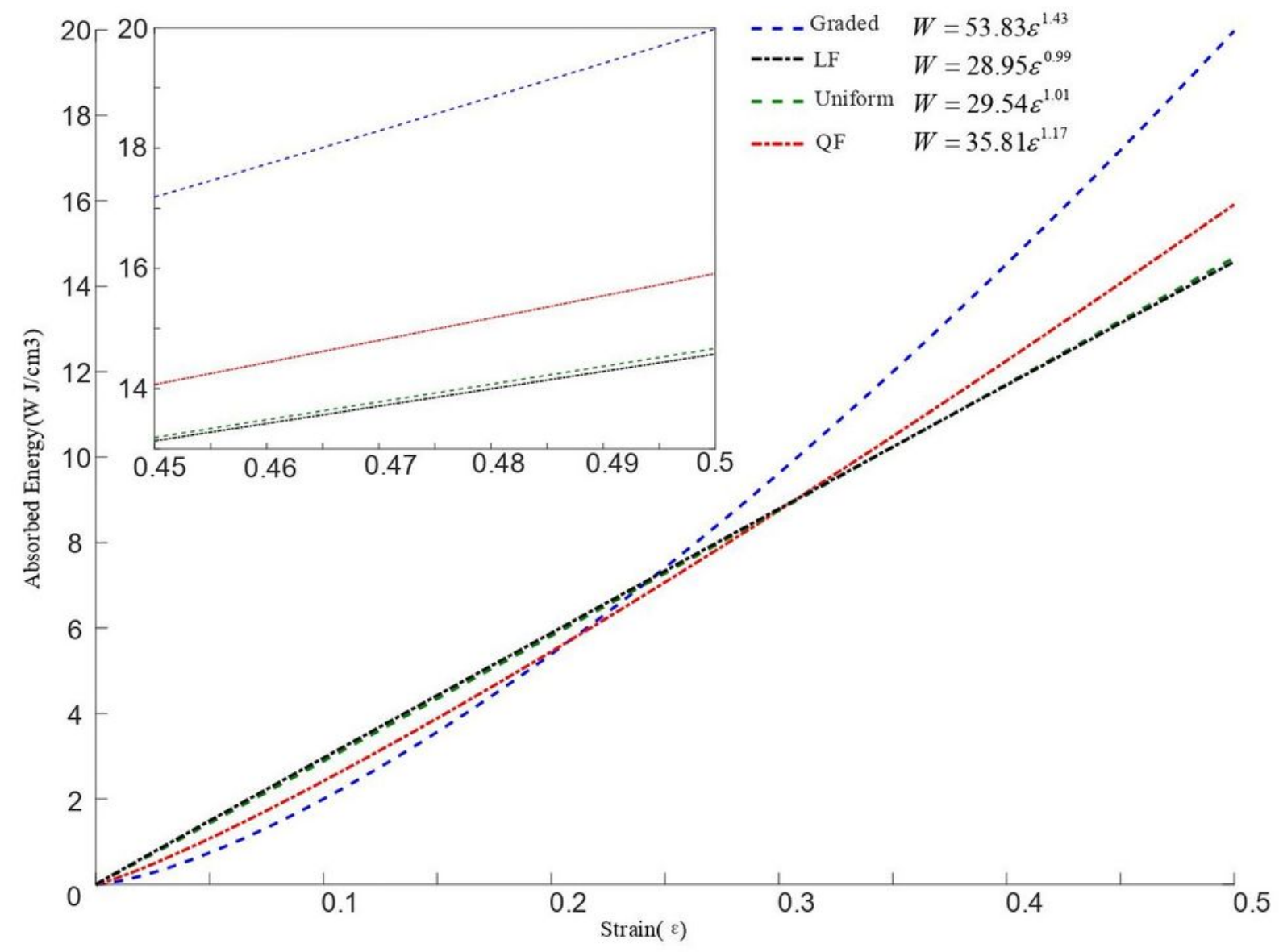

Figure 12

Energy absorption curves of the lattice structures 